\title{
Anmerkungen zur Modellierung und Operationalisierung (allgemeindidaktischer) Unterrichtsplanungskompetenz
}

\author{
Martin Rothland
}

Eingegangen: 16. Juli 2020 / Überarbeitet: 19. April 2021 / Angenommen: 29. April 2021 / Online publiziert: 9. Juli 2021

(C) Der/die Autor(en) 2022

Zusammenfassung Gegenstand des Diskussionsbeitrags ist die im deutschsprachigen Raum prosperierende Forschung zur allgemeindidaktischen Unterrichtsplanungskompetenz. Ausgehend von den Fragen, was allgemeindidaktisch begründet Unterrichtsplanungskompetenz ist, wie sie sich zu den Facetten professioneller Handlungskompetenz von Lehrkräften verhält, wie sie begründet, eigens modelliert und empirisch erfasst wird, kann herausgestellt werden, dass der Diskurs inklusive der vorgeschlagenen Modellierungen und Ansätze der Messung von Unterrichtsplanungskompetenz vielfach von grundlegenden Annahmen geprägt ist, die selbst der empirischen Überprüfung und Absicherung bedürfen. Die diskutierten Modelle und Operationalisierungen allgemeindidaktischer Unterrichtsplanungskompetenz samt einer vorherrschenden Fokussierung auf die Lehrer*innenbildung erzeugen so mitunter mehr offene Fragen als systematisch und differenziert argumentierende theoretische wie empirische Klärungen.

Schlüsselwörter Allgemeine Didaktik · Kompetenz · Kompetenzmodelle · Professionswissen · Unterrichtsplanung 


\title{
Notes on modelling and operationalising (general didactic) lesson planning competence
}

\begin{abstract}
The subject of this contribution is the research on general didactic lesson planning competence that is flourishing in German-speaking research. Starting from the questions what general didactic lesson planning competence is, how it is related to professional competence in general, how it is justified, modelled and empirically explored, it can be shown that the discourse, including the proposed models and approaches to measuring lesson planning competence, is often characterised by fundamental assumptions that themselves require empirical examination and validation. The discussed models and operationalisations of general didactic lesson planning competence together with a predominant focus on teacher education generates partly more open questions than systematic and differentiated argumentative theoretical and empirical clarifications.
\end{abstract}

Keywords General didactics - Teacher competence $\cdot$ Models of teacher competence $\cdot$ Teacher knowledge $\cdot$ Lesson planning

Unterrichtsplanung wird als zentrale Aufgabe von Lehrerinnen und Lehrern beschrieben (vgl. Jäger und Maier 2019; Ziegelbauer und Ziegelbauer 2019). Für das Gelingen von Unterricht als hochkomplexem Prozess gilt sie sogar als Bedingung (Gassmann 2013, S. 105): Eine gute, durchdachte Unterrichtsplanung sei als zentrale Voraussetzung für die Qualität von Unterricht anzusehen (Beck et al. 2008, S. 170; Schnebel et al. 2017, S. 108).

Während in Anleitungen und Ratgebern der Unterrichtsplanung gelegentlich auch ein ,genialisches, intuitives Moment“ anhaftet (Busch 2009, S. 138), gilt im aktuellen, fachübergreifenden Forschungsdiskurs, auf den sich die folgenden Ausführungen beziehen, die Fähigkeit, Unterricht planen zu können, als ,grundsätzlich lehr- und lernbar" (König et al. 2015, S. 377). Die erste und zweite Phase der Lehrer*innenbildung würden in Deutschland dementsprechend auch „,den Schwerpunkt auf die Vermittlung von Planungskompetenz bei angehenden Lehrkräften“ legen (Rey et al. 2018, S. 129), wie sich in den KMK-Standards für die Lehrer*innenbildung in den Bildungswissenschaften konkretisiert, in denen ,explizit Planungskompetenz eingefordert" werde (Weingarten 2019, S. 12). Als Teil bzw. Grundlage dieser Planungskompetenz wird für die erste Phase u. a. ausgeführt, dass die Absolventinnen und Absolventen ,allgemeine und fachbezogene Didaktiken“ kennen (KMK 2019, S. 7). Unterrichtsplanungskompetenz, die es in der ersten Phase der Lehrer*innenbildung den KMK-Standards folgend grundzulegen gilt, wird hier mit der Allgemeinen Didaktik, aber auch mit den Fachdidaktiken, explizit zusammen gebracht. Was aber ist - fachübergreifend und allgemeindidaktisch begründet Unterrichtsplanungskompetenz? Wie verhält sie sich zu den Facetten professioneller Handlungskompetenz gängiger Kompetenzmodelle? Wie wird sie begründet, eigens modelliert und empirisch erfasst?

Diese und weitere Fragen drängen sich umso mehr auf, da neuere Veröffentlichungen wiederholt darauf verweisen, dass es sich bei der (Entwicklung von) 
Planungskompetenz um ein Forschungsfeld handele, dessen Bearbeitung noch am Anfang stehe (Seel und Aprea 2014, S. 5 f.; König et al. 2017a, S. 121): ,empirical research on lesson planning as a skill of preservice teachers is scarce" (König et al. 2020a, S. 801; vgl. König et al. 2020b).

In zweierlei Hinsicht deutet sich hier eine Diskrepanz an: Erstens zwischen der Bedeutung, die der Unterrichtsplanung zugeschrieben wird und der geringen Aufmerksamkeit, die diese so zentrale wie anscheinend wirkmächtige Aufgabe in der Forschung bisher erfahren hat, sowie zweitens zwischen den im Diskurs über die Unterrichtsplanungskompetenz anzutreffenden, als ,sicher' erscheinenden Annahmen und einer unsicheren Forschungs- und Befundlage. Diese bleibt bislang trotz zunehmender Forschungsaktivitäten vor allem auch deshalb unsicher, weil die ihr zugrunde liegenden Modellierungen und Operationalisierungen von allgemeindidaktischer Unterrichtsplanungskompetenz samt der vorherrschenden Fokussierung auf die Lehrer*innenbildung - wie im Folgenden zu zeigen ist - mitunter mehr offene Fragen erzeugen als systematisch und differenziert argumentierende theoretische wie empirische Klärungen zum Forschungsgegenstand bieten.

\section{Hintergrund: Unterrichtsplanung, (Grund-)Prinzipien der Planung und Allgemeine Didaktik}

Der Fokus der Unterrichtsplanung liegt prospektiv auf der dem Unterricht vorgelagerten Planung. Sie gilt zu allererst als ein ,kognitiver Vorgang [...], der selbst wieder kognitive Inhalte, nämlich Wissen, Vorstellungen, Erinnerungen zum Gegenstand hat und dessen Produkt, der Plan nämlich, selbst wieder wirksam werden kann, indem er Teil des Wissens des Lehrers wird“" (Bromme und Seeger 1979, S. 60). Fachübergreifende Grundprinzipien der Unterrichtsplanung und (daraus ableitbare) Kriterien für die Bewertung ihrer Qualität finden sich in der schulpädagogischen Literatur und hier konkret in der Allgemeinen Didaktik bis in die Gegenwart in tradierter Form (vgl. Adl-Amini und Künzli 1980; Kiper und Mischke 2009; Kiel et al. 2014; Standop und Jürgens 2015). Sie zeichnen sich durch ihre Vielfalt und den Anspruch überdauernder Gültigkeit bei gleichzeitiger Offenheit für aktuelle Diskurse etwa zu den Merkmalen/Basisdimensionen der Unterrichtsqualität aus (vgl. Haag et al. 2015; Rothland 2018a) und scheinen selbst nicht durch Forschungsbefunde legitimiert werden zu müssen (zum Empiriedefizit Allgemeiner Didaktik vgl. Bohl 2004; Gräsel 2006; Rothland 2013) ${ }^{1}$. Als die Planung fundierende Unterrichtsprinzipien werden - um einige wenige prominente Beispiele zu geben - generell „Sachgemäßheit, Schülergemäßheit und Zielgemäßheit“" genannt, als regulierende Unterrichtsprinzipien etwa „Anschaulichkeit, Selbsttätigkeit, Motivation“ (Sandfuchs 2006b, S. 697). Wenn es gilt, die allgemeindidaktischen Grundprinzipien der Unterrichtsplanung zu bestimmen, so wird im Handbuch Schulpädagogik (Ziegelbauer und Ziegelbauer

\footnotetext{
1 Die Funktion des hier in Rede stehenden schulpädagogischen Wissens ist trotz der kritisierten mangelnden empirischen Absicherung nicht zu unterschätzen, werden hier doch orientierende Prinzipien und vereinfachende Modelle geboten, die geeignet erscheinen, komplexes Phänomene wie die Unterrichtsplanung überschaubar und womöglich auch handhabbar zu machen (Wolf 2020).
} 
2019, S. 428f.) traditioneller- und bezeichnenderweise allein auf Peterßens Handbuch Unterrichtsplanung (Erstauflage aus dem Jahre 1982; Nachdruck der 9. Auf. 2006) verwiesen. Als Grundprinzipien gelten demnach die (1.) Kontinuität, (2.) Reversibilität, (3.) Eindeutigkeit, (4.) Widerspruchsfreiheit und (5.) Angemessenheit (Peterßen 2006, S. 32-43). Über solche Einzelpublikationen hinaus haben Vogelsang und Riese (2017, S. 54) aus schulpädagogischen Publikationen und Ratgebern die folgenden Kriterien für die Qualität der Unterrichtsplanung abgeleitet: (1.) Stimmigkeit/Interdependenz, (2.) Primat der Ziele, (3.) Variabilität, (4.) Konkretheit/ Kontrollierbarkeit, (5.) Authentizität und (6.) Fachliche Korrektheit. Ergänzend sollen Merkmale der Unterrichtsqualität ,passend zur Thematik und Lerngruppe“ in der Unterrichtsplanung wieder zu finden sein und Hatties Gestaltungsmerkmale für eine hohe Lerneffektivität werden ebenfalls als „Indikatoren der Planungsqualität““ benannt (Werner 2017, S. 82).

Wie sich bereits in den beispielhaft genannten Prinzipien und Kriterien (etwa Primat der Ziele; Interdependenz) andeutet, wird im schulpädagogischen, aber auch im fachdidaktischen Diskurs (Busch 2009) zur Unterrichtsplanung das Planungshandeln u. a. mit der Nutzung oder Anwendung allgemeindidaktischer Modelle verbunden. Traditionell stelle, so eine der Annahmen, die Allgemeine Didaktik ,,den grundlegenden theoretischen Rahmen für die Unterrichtsplanung“ zur Verfügung (Zierer et al. 2015, S. 376; vgl. Bach 2013, S. 37) - und sei es nur als ,didaktisches Gewissen“ im Sinne einer regulativen Idealkonstruktion gut geplanten Unterrichts (Wiater 2006, S. 683).

\section{Unterrichtsplanung als (allgemeindidaktische) Kompetenz?}

Die Bedeutung der Allgemeinen Didaktik für die Unterrichtsplanung manifestiert sich darin, dass „Lehrkräfte über ein spezifisches allgemein- und fachdidaktisches Planungs- und Konzeptionswissen als einer bedeutsamen Facette ihres professionellen Lehrer*innenwissens verfügen“ müssen (Scholl et al. 2019, S. 75), um diese zentrale Aufgabe erfolgreich bewältigen zu können. Dieser Bezug auf das Professionswissen stellt das Bindeglied zur Modellierung professioneller Handlungskompetenz dar, in denen sich das Professionswissens als Kompetenzfacette in Fachwissen, fachdidaktisches Wissen und ein generisches pädagogisches Wissen (pedagogical knowledge, PK) untergliedern lässt. Zu den Dimensionen des generischen pädagogischen Wissens zählen Baumert und Kunter (2006, S. 485) explizit das allgemeindidaktische Konzeptions- und Planungswissen. In unterschiedlichen Definitionen von pädagogischem Wissen finden sich generell allerdings vor allem Bezüge zum interaktiven Unterrichtsgeschehen. Seltener werden Aspekte angesprochen, die sich auf die proaktive Planungsphase beziehen (Voss et al. 2015, S. 192).

Wird Unterrichtsplanung im Modell professionaler Handlungskompetenz von Lehrkräften (Baumert und Kunter 2006) gedacht, dann wäre sie als eine Handlungsanforderung unter vielen anzusehen, deren erfolgreiche und verantwortungsvolle Bewältigung im Sinne des Kompetenzbegriffs von der Ausprägung kognitiver Fähigkeiten und Fertigkeiten (Professionswissen) und dem Zusammenspiel mit motivationalen Orientierungen, Überzeugungen und selbstregulativen Fähigkeiten 
abhängt. Auch wenn die Bezugnahme auf Subdimensionen des Professionswissens eine Einordnung der Fähigkeit, Unterricht zu planen, in das Modell professioneller Handlungskompetenz von Lehrkräften zunächst nahezulegen scheint und explizit benannt wird, dass sie grundsätzlich Teil der professionellen Handlungskompetenz von Lehrkräften sei (Schnebel et al. 2017, S. 108), wird im Forschungsdiskurs unterrichtliche Planungskompetenz in erster Linie jedoch eigenständig definiert, modelliert und operationalisiert. Gesprochen wird von Planungskompetenz (Sandfuchs 2006a, S. 686), unterrichtlicher Planungskompetenz (Gassmann 2013, S. 105) oder auch professioneller Planungskompetenz (Rey et al. 2018, S. 128). Spezielle „Planungskompetenzen bilden die Grundlage für die Gestaltung und Steuerung des Unterrichtsprozesses“ (Kuchartz et al. 2014, S. 20) und Unterrichtplanung wird per se ,als Ausdruck der professionellen Kompetenz von Lehrerinnen und Lehrern“ vorgestellt (Schmaltz 2019, S. 91).

Dabei ist das Konstrukt Unterrichtsplanungskompetenz selbst nicht neu und geht einem kompetenzorientierten Ansatz in der Forschung zum Lehrer*innenberuf und zur Lehrer*innenbildung zeitlich voraus. Bereits Nauck spricht 1996 von einer Planungskompetenz der Lehrkräfte und bestimmt sie wie folgt: Die Schulpädagogik stelle (allgemeindidaktische) Planungsmodelle bereit. „Wer derartige Planungshilfen selbständig und souverän anwendet, gilt als kompetent“ (Nauck 1996, S. 183). So schlicht diese Definition anmutet, die Operationalisierungen in neueren empirischen Studien zur unterrichtlichen Planungskompetenz gehen zumindest zum Teil über dieses Verständnis nicht hinaus (s. Abschn. 4).

Der unterrichtlichen Planungskompetenz werden in aktuellen, komplexeren Annäherungen ihrerseits Kompetenzen bzw. Kompetenzbereiche untergeordnet: (1.) die Fähigkeit, zeitliche Ereignisabfolgen zur erkennen und sinnhaft zu sequenzieren (Weingarten 2019, S. 16f.). Als bedeutsame Kompetenz wird (2.) die Fähigkeit genannt, zur Verfügung stehende zeitliche, materielle und personenbezogene Ressourcen zu berücksichtigen. Unter die personenbezogenen Ressourcen fallen auf Lehrer*innen- und Schüler*innenseite Wissen, Vorkenntnisse Motivation etc. Verknüpft mit diesem zweiten Kompetenzbereich ist die Fähigkeit zur professionellen Diagnose als Entscheidungsgrundlage. Als weitere Kompetenz wird (3.) die Fähigkeit genannt, planerische Alternativen vorzusehen. Ein vierter Kompetenzbereich (4.) bezieht sich auf das Ausmaß des Planungsprozesses, auf die Angemessenheit des Planungsgrads (detailliert vs. planloser Aktionismus) (ebd.). Was traditionell als allgemeindidaktische Grundprinzipien der Unterrichtsplanung galt (s. Abschn. 1), wird nun in Kompetenzen überführt, die Grundlage einer unterrichtlichen Planungskompetenz sind, die ihrerseits Teil der professionellen Handlungskompetenz ist. Kompetenzen überall, so der entstehende Eindruck - wobei ein inflationärer Gebrauch des Kompetenzbegriffs auf verschiedenen Ebenen und eine fehlende Differenzierung von (Teil-)Aspekten professioneller Kompetenz, Kompetenzbereichen und Kompetenzfacetten (Baumert und Kunter 2011) oder Kompetenzklassen, Fähigkeitsdimensionen und Fertigkeiten (Frey 2014) sowie generell von Kompetenz und Performanz (Maag Merki 2009) kein Spezifikum der Forschung zur Unterrichtsplanungskompetenz ist.

Kennzeichnend für den aktuellen Forschungsdiskurs zur Unterrichtsplanungskompetenz ist schließlich generell dessen Verortung in der (Forschung zur) Lehrer*innenbildung. So wird darauf verwiesen, dass ,,der Aufbau von Planungskom- 
petenz zu den zentralen Qualifizierungsaufgaben der Lehrerausbildung " gezählt wird (Seel und Aprea 2014, S. 4; Schnebel et al. 2017, S. 108). Wie aber entsteht Unterrichtsplanungskompetenz?

Eine wiederholt zitierte Antwort gibt Sandfuchs im Handbuch Unterricht (2006a, S. 687; vgl. u. a. Backhaus 2018, S. 46; Seel und Aprea 2014, S. 4): „Planungskompetenz entsteht in einem komplexen biographischen Prozess. Sie setzt sich zusammen aus dem Erwerb theoretischer Erkenntnisse in der Ausbildung, Fort- und Weiterbildung, aus praktischen Erfahrungen in der Berufsausübung, in der das Handlungsrepertoire sowie Routine, d.h. automatisierte Handlungsabläufe, erworben werden. Hinzu kommen persönliche Fähigkeiten vor allem zur Reflexion und zu flexibler Reaktion sowie die professionelle Grundeinstellung" (Sandfuchs 2006a, S. 687). In dieser berufsbiographischen Entwicklung, so eine weitere Annahme, bilden Modelle der Allgemeinen Didaktik eine ,,wichtige Brücke zwischen Theorie und Praxis und können maßgeblich zum Professionalisierungsprozess beitragen“ (Zierer et al. 2015, S. 376). Allgemeindidaktische Unterrichtsplanung wird gar als ein „Kernelement der professionellen Handlungskompetenz“ von Lehrerinnen und Lehrern ausgewiesen (Werner 2017, S. 69) und Unterrichtsplanungskompetenz und allgemeindidaktische (Planungs-)Kompetenz (so Weingarten 2019, S. $132 \mathrm{f}$, kritisch) gleich gesetzt. Letztere bezeichne die für die Planung, Organisation, Durchführung, Analyse und Auswertung von Unterricht, ,erforderlichen allgemeindidaktischen Fähigkeiten und Fertigkeiten, die sich als Anwendung von Wissen über allgemeindidaktische Planungsmodelle von Unterricht beschreiben lassen“ (Bach et al. 2010, S. 158; vgl. auch Schnebel et al. 2017, S. 109).

\section{Diskussion der Modellierung (allgemeindidaktischer) Unterrichtsplanungskompetenz}

Die skizzierten Annahmen zur unterrichtlichen Planungskompetenz „als Anwendung von allgemeindidaktischem Wissen über Planungsmodelle“ (Bach 2013, S. 36), als Zusammenspiel von spezifischem allgemein- und fachdidaktischem Wissen (Scholl et al. 2019, S. 75), spiegeln sich auch in Teilen in den vorgelegten Modellierungen wider. Das Konstrukt Unterrichtsplanungskompetenz wird in den sog. Kompetenzmodellen zudem erweitert - und infolgedessen mitunter auch diffuser.

So liegt den ersten hier aufzugreifenden Modellen die Annahme zugrunde, dass sich in der Unterrichtsplanung „Fachkompetenz, didaktische Kompetenz und pädagogische Kompetenz" manifestieren (Wernke und Zierer 2017, S. 10). Diese seien gleichsam die Pfeiler der Planungskompetenz (ebd.). Ziel sei es, ,anhand theoretisch begründbarer und empirisch abgesicherter Überlegungen die Prozessstruktur zwischen Lehrer, Schüler und Stoff zu gestalten. Hierfür sei eine fachliche, eine pädagogische und didaktische Kompetenz vonnöten - aber auch eine entsprechende Haltung“ (Wernke und Zierer 2017, S. 13).

Um Planungskompetenz zu bestimmen und zu modellieren werden abermals weitere Kompetenzen bemüht. Neben der „Didaktischen Kompetenz“ tritt nun die Fachliche Kompetenz und eine Pädagogische Kompetenz hinzu. Nicht jedoch das Wissen und Können sei ausschlaggebend, sondern - ohne diese Aussage zu belegen - „die 


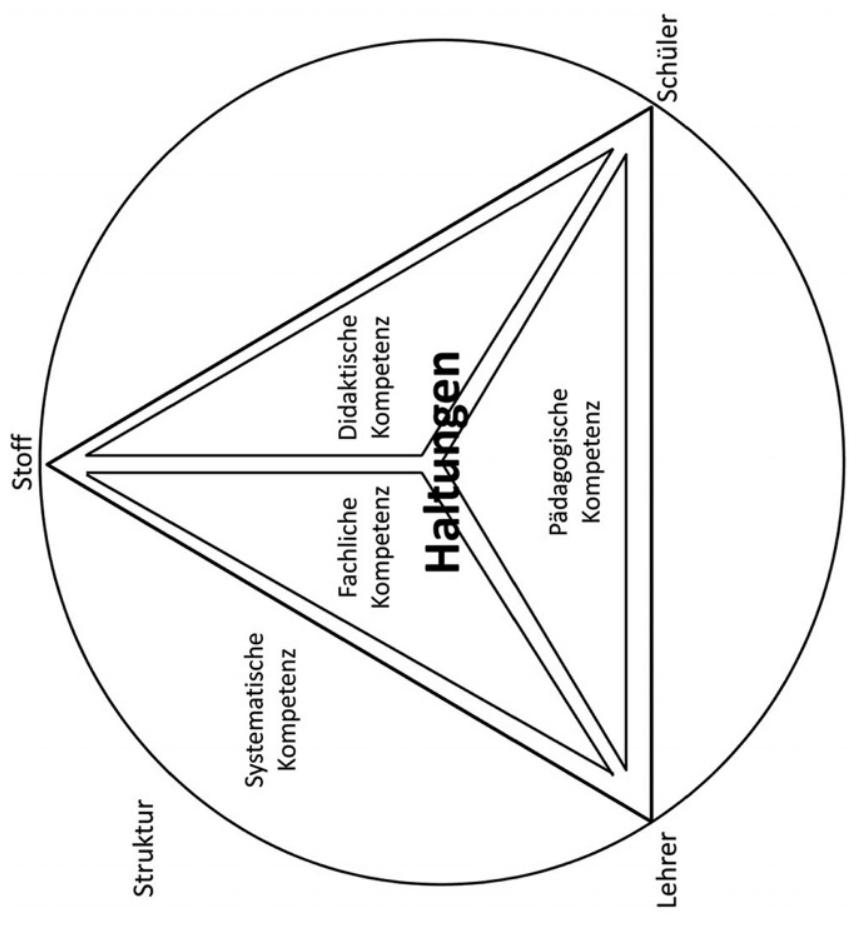

ป 
Tab. 1 Planungskompetenzmodell. (Quelle: Zierer et al. 2015, S. 393)

\begin{tabular}{llll}
\hline & Perspektivität & Dimensionalität & Verständnis \\
\hline Novize & Monoperspektivisch & Eindimensional & Oberflächlich \\
Fortgeschrittener & $\downarrow$ & $\downarrow$ & $\downarrow$ \\
Experte & Multiperspektivisch & Mehrdimensional & Tiefgründig \\
\hline
\end{tabular}

Haltung in Form von Wollen und Werten“ (Wernke und Zierer 2017, S. 11), die „maßgeblich die Planungskompetenz“ beeinflusse (ebd., S. 12; Abb. 1).

Neben dieser Modellierung von Planungskompetenz haben Zierer et al. (2015) ein allgemeindidaktisches Planungskompetenzmodell vorgelegt (Tab. 1). Allgemeindidaktische Planungskompetenz lasse sich demnach anhand der Merkmale Dimensionalität, Perspektivität und Verständnis bestimmen (Werner 2017, S. 71). Sie legen damit ein zweites Planungskompetenzmodell vor, das (1.) in keinen Zusammenhang mit dem Modell der Planungskompetenz im Zentrum des Didaktischen Dreiecks gestellt wird und dessen beanspruchte empirische Herleitung (2.) in Anbetracht der Datengrundlage fragwürdig erscheint. So wurden 68 Lehramtsstudierende im vierten Studiensemester gebeten, einen Planungsentwurf für eine Unterrichtsstunde zu verfassen, wobei ein Teil keine Hilfestellung erhielt und weitere Gruppen eine Hilfe in Form jeweils eines allgemeindidaktischen Modells zu Verfügung gestellt wurde (Zierer et al. 2015, S. 379f.). Wie auf der Basis dieses Settings und der inhaltanalytischen Auswertung von Unterrichtsentwürfen mit und ohne Modell von Studierenden (!) ein Planungskompetenzmodell, das ein Entwicklungsmodell vom Novizen zum Experten sein soll, abgeleitet werden kann, erscheint nicht plausibel.

Planungskompetent, so suggeriert das Modell, ist die Lehrkraft, wenn sie umfassend verschiedene Perspektiven (der Lernenden, der Eltern, der Schulleitung) etc. einnimmt. Es stellt sich die Frage: auf was? Den zu planenden Unterricht? Den Unterrichtsgegenstand? Planungskompetent ist sie des Weiteren, wenn eine Lehrkraft mehrere Phasen berücksichtigt (Planung, Durchführung, Evaluation) und mehrere Dimensionen miteinander verknüpft. Planungskompetent erscheint schließlich die Lehrkraft, die Ziele auf unterschiedlichem Niveau definieren und dazu passende Aufgaben formulieren kann (Zierer et al. 2015, S. 392).

Den beiden Modellen von Zierer et al. ist gemein, dass sie von einer Bezugnahme auf die Modelle professioneller Handlungskompetenz von Lehrkräften absehen. Stattdessen nutzen sie allenfalls Begrifflichkeiten der Kompetenz- und Expertiseforschung. Anders verhält es sich hingegen mit dem schematischen Modell zur Planungskompetenz von König et al. (2017a) als Heuristik (König et al. 2020a), auf das im Forschungsdiskurs vermehrt Bezug genommen wird (so etwa bei Rey et al. 2018; Weingarten 2019; Schmaltz 2019). Planungskompetenz wird hier als kognitive Leistungsdisposition verstanden (König et al. 2015, S. 378) und mit Bezug auf ein Verständnis von Kompetenz als einem Kontinuum von Dispositionen, situationsspezifischen Fähigkeiten und Performanz (Blömeke et al. 2015, S. 7) modelliert. Blömeke et al. (2015) erweitern bisherige Kompetenzmodelle um situations- und verhaltensnahe kognitive Fähigkeiten, die zwischen den kognitiven Disposition als Kompetenzfacetten und hier insbesondere dem Professionswissen einerseits und der Performanz andererseits vermitteln (Abb. 2). In der Adaption des Modells bedingen 


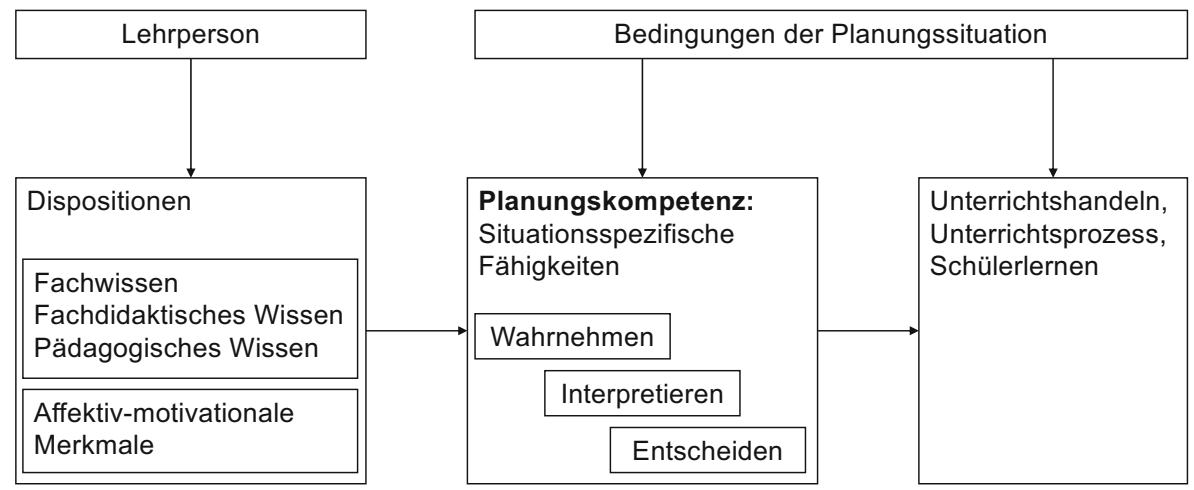

Abb. 2 Schematisches Modell zur Planungskompetenz. (Quelle: König et al. 2017a, S. 125)

Wissensbestände des Professionswissens als kognitive Dispositionen situationsspezifische Fähigkeiten, die - so die Annahme - auch der Planungskompetenz zugrunde liegen. In Rede stehen hier die ,erfolgreiche unterrichtliche Wahrnehmung und Interpretation" bspw. von Lernvoraussetzungen der Schülerinnen und Schüler sowie „erfolgreiche unterrichtliche Entscheidungen“ etwa im Sinne adaptiver Planungsentscheidungen (König et al. 2017a, S. 124). Im Unterrichtsprozess wie auch bei dessen Planung kann gerade das decision making als bedeutende Fertigkeit von Lehrerinnen und Lehrern angesehen werden (Shavelson 1973; Yinger 1980; Westerman 1991).

Wie bei König et al. (2020a, S. 805) benannt ist das schematisches Kompetenzmodell zur Planungskompetenz ein heuristisches, das (1.) bisherige Annahmen und Überlegungen zur unterrichtlichen Planungskompetenz aufgreift, systematisiert und anschlussfähig an die Forschung zur und Modellierung von Kompetenzen von Lehrerinnen und Lehrern macht sowie (2.) zentrale Desiderate ausweist. Auch wenn im aktuellen Forschungsdiskurs zuweilen der Eindruck erweckt wird, dass die im Modell dargestellten Zusammenhänge bereits klar und nachgewiesen wären, so ist (a) der Einfluss und die Relevanz der kognitive Dispositionen (Professionswissen und darin etwa das generische pädagogische Wissen) und der affektiv-motivationalen Merkmale der Lehrerinnen und Lehrer auf die Planungskompetenz ebenso weitgehend empirisch ungeklärt wie (b) die Annahme, dass Planungskompetenz als situationsspezifische Fähigkeit auf der ,professionellen Wahrnehmung“ (professional vision) basiert. Schließlich ist (3.) der Einfluss der Planungskompetenz auf das Unterrichtshandeln, den Unterrichtsprozess und das Schüler*innenlernen zu überprüfen. ${ }^{2}$

Der Ansatz, Kompetenz als ein Kontinuum von Dispositionen und situationsspezifischen Fähigkeiten zu fassen, gerät allerdings im Zuge dieser Modellierung etwas in den Hintergrund. Während sich die bisherigen Modelle professioneller Handlungskompetenz auf die Kompetenzfacetten und die Annahme ihrer wechselseitigen

\footnotetext{
2 Fokussiert auf den Aspekt der didaktischen Adaptivität trägt derzeit die Forschungsgruppe um König (König et al. 2015, 2020a, b) zur anteiligen Bearbeitung dieser Forschungsdesiderate (insbesondere zu a und b) bei.
} 
Bedingtheit konzentrierten, die als Dispositionen der Lehrerinnen und Lehrer bei Blömeke et al. (2015) sowie König et al. (2017a) bezeichnet werden, werden diese Kompetenzfacetten nun in der Adaption der Planungskompetenz als situationsspezifischer Fähigkeit zugrunde gelegt. Sind sie damit in ihrem Zusammenspiel nicht mehr selbst als professionelle Handlungskompetenz, sondern als Dispositionen für Kompetenz zu verstehen? Und was wäre dann die Performanz? Das Unterrichtshandeln, der Unterrichtsprozess und das Schüler*innenlernen, wie Blömeke et al. (2015) nahelegen, oder die (adaptiven) Planungsentscheidungen, die bspw. beim Abfassen eines schriftlichen Unterrichtsentwurfs getroffen und niedergeschrieben werden? Schließlich wären doch das Wahrnehmen, Interpretieren und Entscheiden eher als Performanz denn als Kompetenz als latentem Konstrukt, als kognitiver Disposition anzusehen, wenn die Differenz von Kompetenz und Performanz ernst genommen wird (vgl. Maag Merki 2009). Eine in diesem Sinne korrigierte und infolgedessen an die Forschung zur generischen Handlungskompetenz von Lehrerinnen und Lehrern anschlussfähige Relationierung legt die Modellierung in König et al. (2020a, S. 805) nahe, in der nicht von Planungskompetenz, sondern von Unterrichtsplanung als Teil der Kompetenz von Lehrkräften gesprochen wird. ${ }^{3}$

\section{Diskussion der Operationalisierung und empirischen Erfassung (allgemeindidaktischer) Unterrichtsplanungskompetenz}

Wenn es gilt, in der neueren Forschung die (allgemeindidaktische) unterrichtliche Planungskompetenz empirisch zu erfassen, so kommen unterschiedliche Zugänge zur Anwendung: die Erhebung über Selbsteinschätzungen (4.1), ein Wissenstest (4.2) oder die kategorienbasierte inhaltsanalytische Untersuchung von Produkten der Unterrichtsplanung (4.3), also von Zeugnissen der Performanz wie etwa schriftlichen Unterrichtplanungsentwürfen. Tab. 2 bietet zunächst eine Übersicht über die im Folgenden berücksichtigen Instrumente.

Vor der Diskussion der in Tab. 2 aufgeführten unterschiedlichen Operationalisierungen unterrichtlicher Planungskompetenz ist zu betonen, dass diese - bei allen kritischen Einlassungen, die im Folgenden zur Diskussion gestellt werden - allesamt wertvolle und relevante Bemühungen verbunden mit zum Teil erheblichen Forschungsanstrengen darstellen, um Planungskompetenz empirisch zu fassen und abzubilden. Dies gilt für den Versuch, handhabbare, mit geringem Zeitaufwand bearbeitbare und zugleich aussagekräftige Selbsteinschätzungsskalen zu konstruieren und zu evaluieren. Es gilt ebenso für das schwierige Geschäft der Testentwicklung, der, wie beispielsweise im Falle des sieben Vignetten umfassenden Tests zu ,Erfassung allgemeindidaktischer Planungskompetenz" von Schüle et al. (2017; Abschn. 4.2),

\footnotetext{
3 Eine Adaption des Modells von König et al. (2017), das die Unterrichtsplanungskompetenz als professionelle adelt und eine Rahmung mittels des strukturtheoretischen Professionsansatzes und des berufsbiographischen Ansatzes versucht, potenziert hingegen die Beliebigkeit der Begriffsverwendung, in dem hier Dispositionen Grundlage der Planungskompetenz sind, die ihrerseits das Unterrichtshandeln und den Unterrichtsprozess als Handlungskompetenz bedingen (Schmaltz 2019, S. 103). Was ursprünglich die Performanz war, wird nun zur Kompetenz.
} 


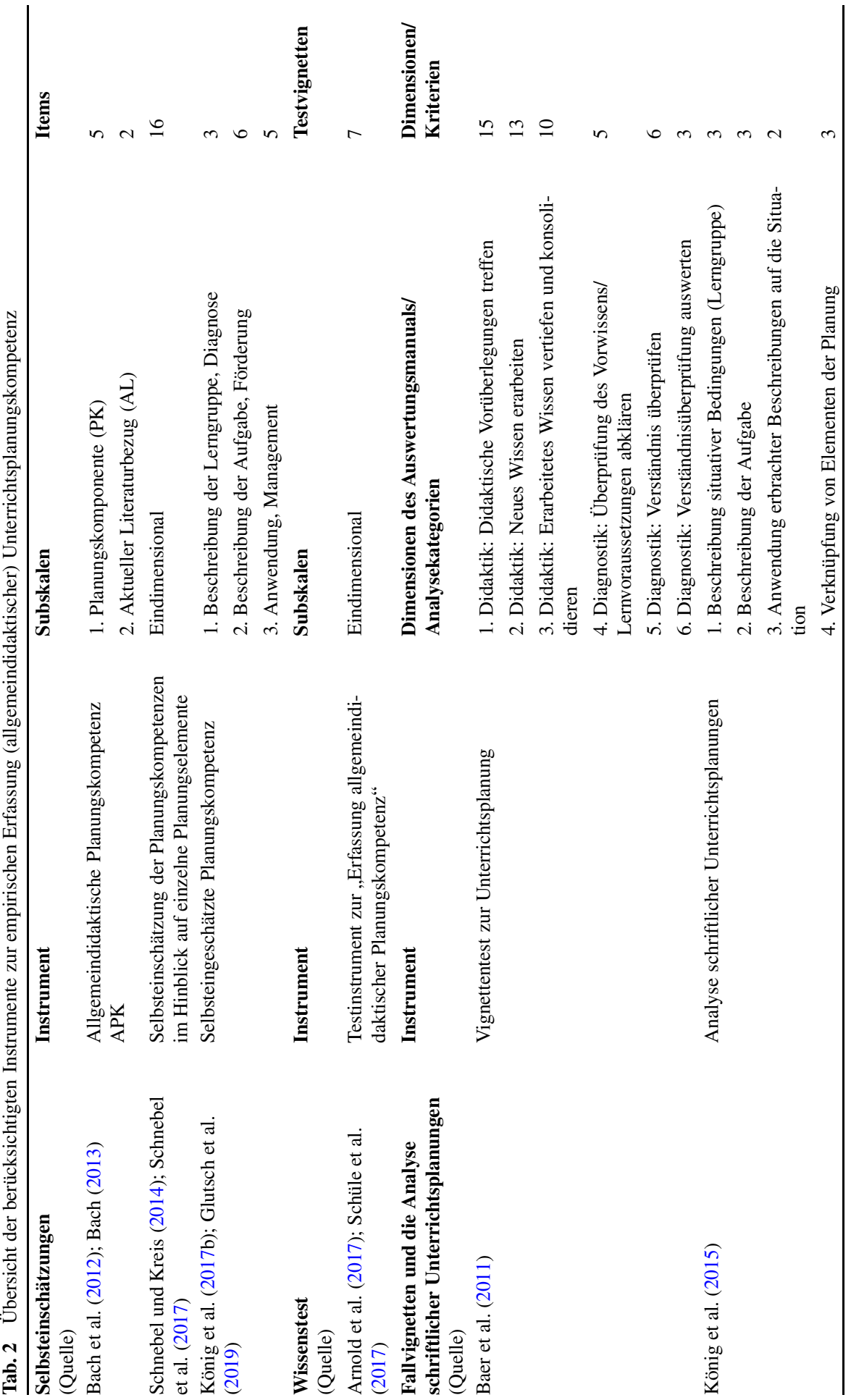


die Entwicklung und aufwändige empirische Prüfung von 26 Testvignetten voraus geht.

\subsection{Selbsteinschätzungen der Planungskompetenz}

Die allgemeindidaktische Unterrichtsplanungskompetenz wird im Forschungsdiskurs u.a. über die Selbsteinschätzung ausgewählter Fähigkeiten und Fertigkeiten durch angehende Lehrkräfte erhoben. Entwickelt wurden die Skalen zur Erfassung Allgemeindidaktische[r] Planungskompetenz (APK) (Bach et al. 2010; Bach 2013), die Skala zur Erhebung der Selbsteinschätzung der Planungskompetenzen im Hinblick auf einzelne Planungselemente (Schnebel und Kreis 2014; Schnebel et al. 2017) sowie die Skalen zur Selbsteingeschätzten Planungskompetenz (König et al. 2017b; Glutsch et al. 2019; s. Tab. 3).

Mit der ersten Dimension der APK-Skala, der Planungskomponente (5 Items), werden, so der Anspruch, zentrale Aspekte großer allgemeindidaktischer Planungsmodelle operationalisiert. Hinzu kommt mit ,aktueller Literaturbezug“ eine zweite Dimension (2 Items). Hier soll die Verwendung von Literatur bei der Unterrichtsplanung erfasst werden, wobei die Nutzung von allgemeindidaktischer und fachdidaktischer Literatur eine ,gemeinsame Fähigkeitskomponente“ abbilde (Bach 2013, S. 159). Die APK-Skala spiegele in der zweidimensionalen Struktur ,die allgemeindidaktischen Modelle wider" (Bach et al. 2012, S. 116).

In der Studie von Schnebel et al. (2017) wird Planungskompetenz anhand einer eindimensionalen Skala mit 16 Items darüber erfasst, wie Studierende ihre Kompetenzen in der „Ausarbeitung zentraler Planungsdimensionen Ziele, Lerninhalte, fachspezifische Arbeitsformen, curriculare Verortung, methodische Entscheidungen, Lernvoraussetzungen und diagnostische Aktivitäten sowie kognitiv anregende Aufgabenstellungen“ einschätzen (ebd., S. 109f.). In der Zusammenschau erscheint unterrichtliche Planungskompetenz in dieser Operationalisierung als Ansammlung verschiedener Fähigkeitsdimensionen, die ihrerseits zum Teil hierarchisch zu strukturieren wären, da sie auf unterschiedlichen Ebenen liegen bzw. sich zum Teil bedingen (,Ich informiere mich über die Lernvoraussetzungen der Schüler“ $\rightarrow$ „Unterschiedliche Lernvoraussetzungen berücksichtigen“ $\rightarrow$,,Vorwissen der Lernenden berücksichtigen"= eine Facette der Lernvoraussetzungen).

Die Ausrichtung der dreidimensionalen Skala Selbsteingeschätzte Planungskompetenz erscheint bezogen auf ihre inhaltliche Ausrichtung konzentrierter. Sie fokussiert mit den ersten beiden Dimensionen auf Aspekte der Adaptivität als bedeutender Facette der Unterrichtsplanung (vgl. Scholl et al. 2019, S. 86): (1.) die Diagnose der Lerngruppe (,diagnosis of students“) und (2.) der davon abhängigen Aufgabenauswahl und -formulierung (,design of tasks“). Hinzu kommen (3.) darüber hinausgehende Planungsaspekte (,,design of the learning process“) (König et al. 2017b, S. 403), die zum Teil auch in den beiden zuvor angesprochenen Selbsteinschätzungsskalen aufgegriffen werden.

Es zeigen sich damit zum einen deutliche Überschneidungen in der empirischen Erfassung generischer Unterrichtsplanungskompetenz mittels Selbsteinschätzungen (etwa Lernvoraussetzungen der Schülerinnen und Schüler diagnostizieren und berücksichtigen) und zum anderen nur einmalig auftretende Facetten (bspw. Transfer 


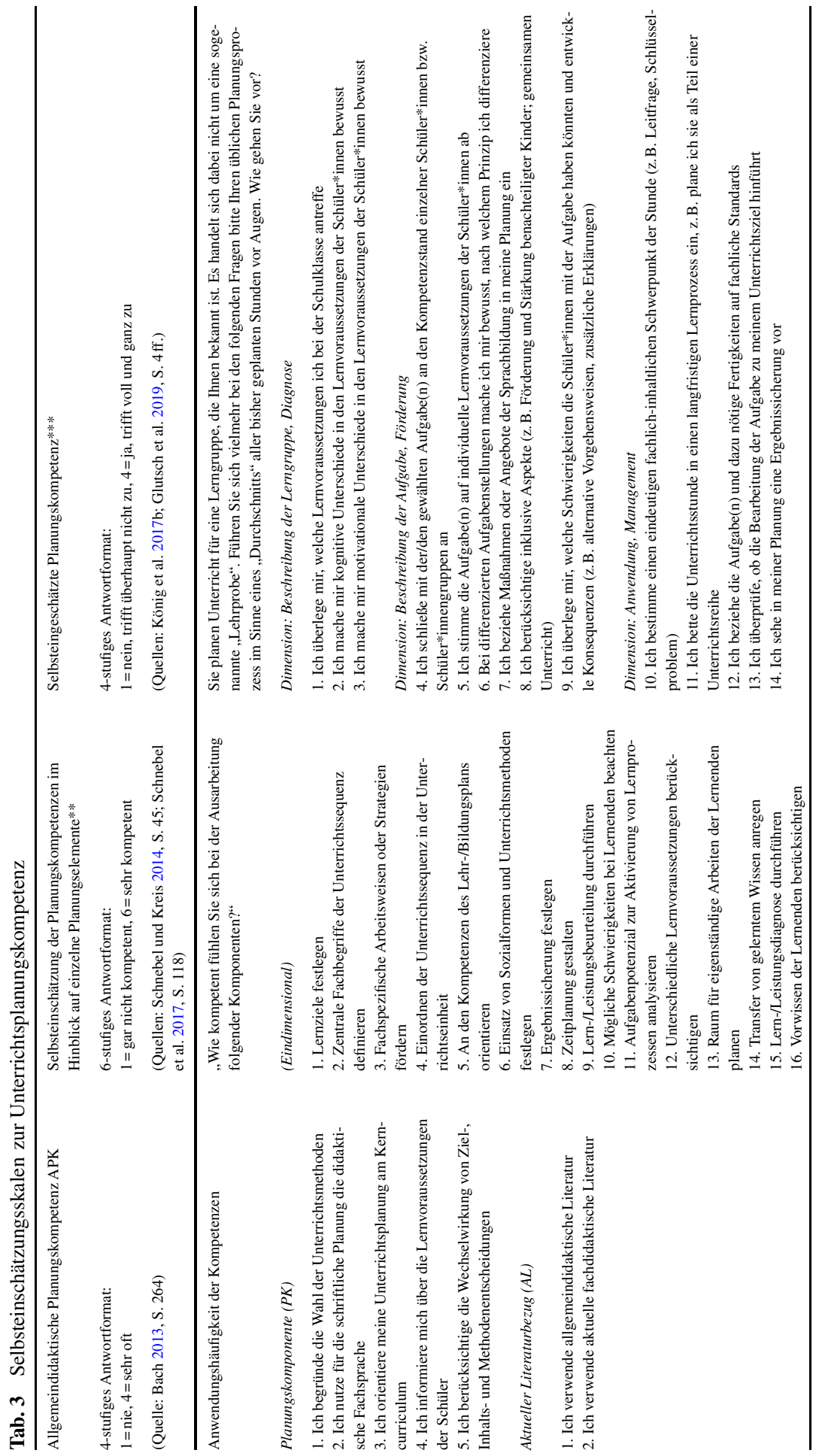


erworbenen Wissens oder die Bedeutung von Aufgaben für die Zielerreichung des geplanten Unterrichts). Welche Aspekte machen aber die Unterrichtsplanungskompetenz aus? Eine befriedigende Antwort auf diese Frage scheint über das Studium der Selbsteinschätzungsskalen (noch) nicht recht auffindbar.

Generell korrespondieren die angeführten Operationalisierungen der Planungskompetenz mit den im Abschn. 1 angesprochenen allgemeindidaktisch inspirierten (Grund-)Prinzipien der Unterrichtsplanung. Sie stimmen mit dem skizzierten allgemeindidaktischen Diskurs zur Unterrichtsplanung und zur Planungskompetenz insbesondere im Falle der Allgemeindidaktischen Planungskompetenz (APK) insofern überein, als dass sie empirisch abgesicherte Erkenntnisse als Voraussetzung für die Legitimation der Itemformulierungen voraussetzen, die nicht vorliegen, so etwa die Annahme, dass schriftliche Unterrichtsplanungen dann qualitativ hochwertiger seien und einen besseren Unterrichtsprozess, ein besseres Unterrichtsangebot bedingen würden, wenn sie sich (häufiger) einer didaktischen Fachsprache bedienen. Dabei ist auch grundsätzlich nicht geklärt, ob und in welchem Maße schriftliche Unterrichtsplanungen überhaupt relevant für die Erklärung der Qualität des durchgeführten Unterrichts sind. Planungskompetent(er) ist der APK Skala folgend überdies der Lehramtsstudent/die Lehramtsstudentin, der/die häufiger die Auswahl der Unterrichtsmethoden begründet und häufiger allgemeindidaktische Literatur verwendet bzw. der/die Lernziele oder eine Ergebnissicherung festlegt etc. Sie erschöpft sich in dieser Art der Erfassung in der Deskription einer Auswahl von Fertigkeiten, die keine Rückschlüsse darüber zulässt, wie die Qualität der Unterrichtsplanung und damit der Grad der Ausprägung der Planungskompetenz zu bestimmen ist.

\subsection{Wissenstest: Planungskompetenz als allgemeindidaktisches Planungswissens}

Ein Testinstrument zur „Erfassung allgemeindidaktischer Planungskompetenz“ haben Schüle et al. (2017, S. 17) vorgelegt. Dieser „Kompetenztest“" erweist sich als Wissenstest, der mit insgesamt sieben Testvignetten beansprucht, allgemeindidaktisches Planungswissens von (angehenden) Lehrpersonen zu erfassen. Was allgemeindidaktisches Planungswissens und damit Voraussetzung der Unterrichtsplanung sein soll, das kann am Beispiel von zwei Testvignetten illustriert werden (Tab. 4).

Bereits das erste Beispiel wirft die Frage auf, ob es sich hier um (Unterrichts-)Planungswissen handelt, oder nicht vielmehr um Wissen über Klafkis Bildungsverständnis als Zusammenhang der drei genannten Grundfertigkeiten. Darüber hinaus erscheint die kritische Anfrage entscheidend für die Legitimation eines solchen Testitems, welche Bedeutung dieses Wissen über Klafkis Bildungsverständnis erwiesenermaßen für die Bewältigung der Aufgabe, Unterricht erfolgreich zu planen, und für die Qualität der Unterrichtsplanung hat.

Bezogen auf das zweite Beispiel (Tab. 5) stellen sich dieselben grundsätzlichen Fragen: Ist es (Unterrichts-)Planungswissen oder „Reflexionswissen“", das hier getestet wird? Und welche Bedeutung hat dieses Reflexionswissen empirisch nachprüfbar für die (Qualität der) Unterrichtsplanung?

Bereits die Validierung des Testinstruments unterstützt die angeführten Rückfragen zur Bedeutung des getesteten Planungswissens für das Gesamtkonstrukt 
Planungskompetenz, ist der statistische Zusammenhang zwischen allgemeindidaktischem Planungswissen und der selbsteingeschätzten allgemeindidaktischen Planungskompetenz (APK) mit $r=0,31$ ( $S E=0,11, p<0,01)$ eher überraschend gering. Schüle et al. (2019, Folie 22) verweisen generell selbst auch einschränkend darauf, dass die ,empirische Bedeutsamkeit der gewählten allgemeindidaktischen Ansatzpunkte von Heimann et al. (1965) sowie Klafki $(1958,1980)$ [...] trotz des entwickelten Testverfahrens weiterhin ungeklärt" bleibe.

$\mathrm{Zu}$ fragen wäre darüber hinaus, woher das Wissen, das mit dem vorgelegten Instrument getestet wird, stammt. Erneut wird hier wie in weiteren Teilen des gesamten Forschungsdiskurses zur Unterrichtsplanungskompetenz von einer starken, aber bislang nicht empirisch unterstützten Annahme ausgegangen, nämlich dass „Allgemeindidaktische Planungsmodelle [...] nach wie vor ein zentrales Element der universitären Lehrerbildung“" seien (Schüle et al. 2017, S. 17; vgl. Bach et al. 2010, S. 158). Werden hier Studien zur Gestaltung der Curricula für den Bereich Bildungswissenschaften an deutschen Universitäten zurate gezogen, so zeigen sich etwa für das Wintersemester 2010/2011 an 16 ausgewählten Hochschulen (je Bundesland eine) beträchtliche Unterschiede (Hohenstein et al. 2014). Die Ergebnisse der Analyse machen allerdings für den Bereich Unterrichten auch deutlich, dass das Thema „Didaktik/Unterrichtsplanung“ von 15 der 16 Hochschulen in den Curricula berücksichtigt wird (ebd., S. 502). Es wäre allerdings vorschnell, aus der Verankerung des Kompetenzbereichs zu schließen, dass konkret ,Allgemeindidaktische Planungsmodelle [...] nach wie vor ein zentrales Element der universitären Lehrerbildung“ darstellen (Schüle et al. 2017, S. 17). Auch im Kompetenzbereich Unterrichten findet sich in der inhaltlichen Ausgestaltung ein hoher Grad der Differenzierung (vgl. Schulze-Stocker 2016; Lohmann et al. 2011). Und das intendierte Curriculum muss bekanntlich nicht mit dem implementierten Curriculum übereinstimmen. Dass das hier getestete Wissen womöglich sogar unabhängig von den Angeboten der ersten Phase der Lehrer*innenbildung sein könnte, deutet sich an, wenn sich im Zuge der Überprüfung der Kriteriumsvalidität zeigt, dass sich das jeweils erzielte Ergebnis des Testinstruments zur „Erfassung allgemeindidaktischer Planungskompetenz“ als unabhängig von der Fortdauer des Studiums erweist (Schüle et al. 2019).

\subsection{Fallvignetten und die Analyse schriftlicher Unterrichtsplanungen}

Das Wissen zur Planung von Unterricht wird in der Forschung zur Planungskompetenz auch über Vignetten als sprachlich repräsentierte Problemsituationen erfasst

Tab. 4 Testvignette 1. (Quelle: Arnold et al. 2017, S. 4)

Beispiel 1
Sie sind an der Fächerübergreifenden Unterrichtseinheit (Deutsch, Politik, Mathematik, Kunst) zum The-
ma „Imagewerbung“ beteiligt und planen eine Analyse von Anzeigen großer Stromkonzerne. Mit wel-
chem/n der aufgeführten Begriffe lässt sich allgemeindidaktisch begründen, dass die Schüler die Haus-
haltstarife, die Bedingungen für einen Anbieterwechsel und den Anteil von regenerativen Energiequellen
vergleichen? Befähigung zu zunehmender
Antwortalternativen Selbstbestimmung ${ }^{\mathrm{a}}$, Solidarität $^{\mathrm{a}}$, Autonomie, Mitbestimmung $^{\mathrm{a}}$

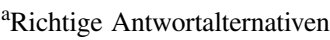


Tab. 5 Testvignette 2. (Quelle: Arnold et al. 2017, S. 8)

Beispiel 2

Nachdem Sie Ihre erste Unterrichtsstunde gehalten haben, analysieren Sie gemeinsam mit Ihrem Mentor die Stunde. Welche Frage/n wäre/n allgemeindidaktisch bedeutsam?

Antwortalternativen 1. An welchen Stellen gab es Abweichungen vom geplanten Unterrichtsverlauf?

2. Wie war die Akzeptanz der Schüler bezüglich meines Auftretens als Lehrperson?

3. Waren die Schüleraktivitäten in der geplanten Stunde auf die Lernziele ausgerichtet? ${ }^{\mathrm{a}}$

4. Haben die Schüler die von mir intendierten Lernziele der Unterrichtsstunde erreicht? ${ }^{\mathrm{a}}$

${ }^{\text {a}}$ Richtige Antwortalternativen

Tab. 6 Eingesetzte Vignetten. (Quelle: Baer et al. 2011, S. 96)

\begin{tabular}{ll}
\hline Vignette 1 & „David Wagner ist beim Vorbereiten von Unterricht immer wieder unsicher, ob die Schü- \\
& lerinnen und Schüler die zentralen Lernziele erreichen. Besonders im Bereich Natur und \\
& Technik zweifelt er daran, ob seine Vorbereitungen professionell genug sind. Schildern Sie \\
& ihm, wie er bei der Unterrichtsvorbereitung in Natur und Technik vorgehen könnte. Gehen \\
& Sie bitte auf sämtliche Vorbereitungsschritte ein und begründen Sie diese.“ \\
Vignette 2 & "Simone Landolt hat in der Ausbildung gehört, dass eine gute Diagnose des Lernstandes \\
& der Schülerinnen und Schüler für die Planung und Steuerung des Unterrichts wichtig sei. \\
& Simone möchte deshalb regelmäßig während der Lektion im Bereich Natur und Technik das \\
& Verstehen der Schülerinnen und Schüler diagnostizieren können. Erklären Sie bitte Simone, \\
& wie sie dies schon bei der Vorbereitung berücksichtigen kann und wie sie das Ergebnis \\
& der Diagnose für die Steuerung und die weitere Planung des Unterrichts nutzen könnte. \\
& Begründen Sie Ihre Antwort.“
\end{tabular}

(Baer et al. 2011, S. 96). Auf der Basis des im Folgenden vorgestellten Vignettentests, der bei Lehramtsstudierenden im Rahmen von Praktika und zudem im Berufseinführungsjahr zur Anwendung kam, wird beansprucht, die Kompetenz, Unterricht zu planen, abbilden zu können (Larcher et al. 2010, S. 67; Tab. 6).

Eine inhaltsanalytische Auswertung der Bearbeitung der Vignetten erfolgte anhand der Dimensionen (1.) Sachkompetenz, (2.) didaktische Kompetenz, (3.) diagnostische Kompetenz und (4.) Klassenführungskompetenz. Gesprochen wird von Kompetenzbereichen oder Dimensionen der Planungskompetenz (Larcher et al. 2010, S. 63, 69) und nicht allein vom Wissen zur Unterrichtsplanung (Baer et al. 2011, S. 96). Zu den Indikatoren der Dimensionen Klassenführung und Sachkompetenz wurden über verschiedene Messzeitpunkte hinweg von den Studierenden nur sehr wenige Aussagen gemacht, sodass diese in den Auswertungen nicht weiter berücksichtigt wurden (Baer et al. 2011, S. 99).

Was mit Blick auf die Operationalisierung allgemeindidaktischer Planungskompetenz insbesondere die hier angeführte didaktische Kompetenz ist oder ausmacht, kann anhand der Dimensionen des Auswertungsmanuals nachvollzogen werden. Das Kategoriensystem der Untersuchung umfasst insgesamt 56 Verhaltensindikatoren. Werden die in Tab. 7 aufgeführten Aspekte in der Bearbeitung einer Vignette benannt, wird er bei der ersten Nennung mit einem Punkt gewertet. Die Höhe der Punktzahl, so die Annahme, bilde die „Differenziertheit der Unterrichtsplanung und damit eine Facette der Planungskompetenz" ab (Kuchartz et al. 2014, S. 22). Nicht allein Facette der Planungskompetenz, sondern auch Qualitätskriterium der Unter- 
Tab. 7 Dimensionen des Auswertungsmanuals mit Kriterien und Indikatoren. (Quelle: Baer et al. 2011, S. 97 f.)

Didaktik (38 Dimensionen)

A Didaktische Vorüberlegungen treffen

- Langfristige Planung von Unterrichtseinheiten, damit Zeit für die Vorbereitung bleibt (z. B. förderliche Lernprozesse)

- Ausrichtung des Unterrichts auf den Lehrplan

- Ausrichtung des Unterrichts auf Lernziele

- Erstellen eines Zusammenhanges zwischen den Themenbereichen

- Planung von Bezügen innerhalb der Unterrichtseinheit/en (z. B. Übergänge von Lektionen; , roter Faden“ in Lektion)

- Bezugnahme auf didaktische Theorien

- Reflexion der Planungsideen mit anderen Fachleuten (Themenwahl, Vorgehensweise, ...)

- Effektive und schonungsvolle Nutzung der eigenen Ressourcen

- Auswahl von Materialien

- Auswahl verschiedener Medien

- Ausrichtung des Unterrichts in Hinblick auf die Motivation

- Einschätzung der Lernzeit

- Vorgängiger Testlauf der Unterrichtseinheit (z. B. Versuche)

- Reflexion des Unterrichts durch die LP

- Rückkoppelung der Unterrichtsnachbereitung für folgende Lektionen

$B$ Neues Wissen erarbeiten

- Einsatz verschiedener Methoden

- Auswahl geeigneter Sozialformen (Gruppenunterricht, Partnerarbeit, ...)

- Ermöglichung von Lernen in verschiedenen Bereichen (kognitiv, kreativ, affektiv, sozial, multimodal)

- Offenlegung der Lernziele gegenüber den S

- Anregung der S zu Vermutungen

- Ermöglichung konkreter Handlungserfahrungen der S

- Verbindung zu einem theoretischen Model

- Bezugnahme zu aktuellen Ereignissen oder Alltagserlebnissen der S

- Erstellung von Zusammenhängen und Initiierung von Vernetzungen

- Vermittlung von Arbeitstechniken und Lernstrategien

- Führung von Lerntagebüchern durch S

- Aufnahme der Fragestellungen der S

- Unmittelbare Anpassung der Planung an das Unterrichtsgeschehen
C Erarbeitetes Wissen vertiefen und konsolidieren

- Anregung der kognitiven Aktivität der S (Wissenstransfer, Umsetzung, aktive Mitarbeit)

- Ermöglichung der freien Verarbeitung des Themas (z. B. Projektarbeit) durch die S

- Schriftliche Fixierung der wichtigsten Erkenntnisse mit der Klasse

- Individuelle schriftliche Fixierung der Erkenntnisse durch die S

- Darbietung des neuen Wissens durch die S

- Planung von Übungsphasen zur Vertiefung des neuen Wissens

- Planung von Repetitionen bei mangelndem Verständnis

- Differenzierung des Unterrichts, um Fähigkeiten und Interessen der $\mathrm{S}$ gerecht zu werden

- Hilfestellung bestimmter S durch einzelne S

- Einsatz von Lernpartnerschaften (Tandemlernen)

Diagnostik (13 Dimensionen)

A Überprüfung des Vorwissens/Lernvoraussetzungen abklären

- Abklärung des außerschulisch erworbenen Vorwissens

- Abklärung des schulisch erworbenen Vorwissens

- Abklärung der Interessen der S

- Abklärung der Denk-/Lernstile der S

- Abklärung der sozialen Voraussetzungen (z. B. Klassenklima)

$B$ Verständnis überprüfen

- Beobachtung der Mimik und Gestik (nonverbales S-Verhalten)

- Stellen von Verständnisfragen

- Aufforderung an $\mathrm{S}$, das neue Wissen mündlich in eigene Worte zu fassen

- Schriftliche Abfrage des Verständnisses

- Aufforderung an S, das neue Wissen schriftlich in eigene Worte zu fassen

- Anregung der S zur Selbstevaluation des Lernens

C Verständnisüberprüfung auswerten

- Summative Überprüfung (dient Kontrolle)

- Formative Überprüfung (dient Rückmeldung)

richtsplanung ist hier in dieser Form der empirischen Erfassung der Grad der Differenzierung. Dass aber ein maximaler Differenzierungsgrad mit der Güte der Unterrichtsplanung und darüber hinaus mit der Qualität des anschließend durchgeführten Unterrichtsprozesses zusammenhängt, auch dies gilt es zunächst forschungsbasiert zu belegen. Angenommen werden könnte schließlich auch, dass eine Maximierung der Differenzierung im Sinne einer „Überplanung“ kontraproduktiv ist.

Ein Kategoriensystem zur Analyse schriftlicher Unterrichtsplanungen im Referendariat, das ebenfalls Rückschlüsse auf eine Konkretisierung dessen ermöglicht, was unterrichtliche Planungskompetenz ausmacht, haben König et al. (2015, S. 386) vorgelegt. Konzeptueller Hintergrund ist das in Abschn. 3 vorgestellte schematische Modell zur Planungskompetenz (König et al. 2017a), wobei die hier in Rede stehenden Analysekriterien insbesondere die unterrichtliche Wahrnehmung und Interpretation etwa von Lernvoraussetzungen der Schülerinnen und Schüler adressieren (Tab. 8). 
Diagnostische und allgemeindidaktische Kriterien werden hier in einer aufeinander aufbauenden Abfolge gedacht und systematisch aufeinander bezogen, während sie im vorhergehenden Vignettentest (abgesehen von der Unterteilung in die Unterkategorien A, B und C) und auch im Rahmen der Erfassung der Selbsteinschätzung der Planungskompetenzen im Hinblick auf einzelne Planungselemente (Schnebel und Kreis 2014) als Sammlung aufgeführt werden. Der Fokus ist bei König et al. (2015) allerdings auch konzentriert auf die Abstimmung der Aufgabenstellung in der Erarbeitungsphase der geplanten Unterrichtsstunde auf die Lerngruppe. Explizit wird deutlich gemacht, dass ein spezifischer Ausschnitt von Planungskompetenz (Passung von Lehrangebot und Lerngruppe) in den Blick genommen wird, während in den zuvor vorgestellten Operationalisierungen zumindest der Anspruch deutlich umfassender erscheint.

\section{Bilanz: Herausforderungen und Forschungsperspektiven}

Wie in der vorhergehenden Zusammenschau kenntlich gemacht werden konnte, ist der deutschsprachige Diskurs zur (allgemeindidaktischen) unterrichtlichen Planungskompetenz inklusive der Überlegungen zu deren Modellierung sowie der Ansätze zu ihrer Operationalisierung vielfach geprägt von grundlegenden Annahmen, die, obwohl sie der empirischen Überprüfung und Absicherung in der Mehrzahl der Fälle bedürfen, als gesicherte Einsichten bzw. als Tatsachen präsentiert werden. ${ }^{4}$ $\mathrm{Zu}$ diesen zählen beispielsweise die Vermutungen, (1.) dass die Qualität der Unterrichtsplanung die Qualität des Unterrichts bedingt, (2.) dass der Schwerpunkt der Lehrer*innenbildung auf der Vermittlung von Planungskompetenz liegt, (3.) dass die Allgemeine Didaktik ,den grundlegenden theoretischen Rahmen für die Unterrichtsplanung“ zur Verfügung stellt (Zierer et al. 2015, S. 376), (4.) dass allgemeindidaktische Unterrichtsplanung ein „Kernelement der professionellen Handlungskompetenz" von Lehrerinnen und Lehrern ist (Werner 2017, S. 69), (5.) dass allgemeindidaktische Kompetenz ,als Anwendung von allgemeindidaktischem Wissen über Planungsmodelle" zu verstehen ist (Bach 2013, S. 36; Bach et al. 2010, S. 158) oder (6.), dass ein maximaler Differenzierungsgrad mit der Güte der Unterrichtsplanung zusammenhängt. Angesichts dieser und weiterer Gültigkeit beanspruchender Annahmen stellt sich - um ein Beispiel herauszugreifen - die Frage, wann von wem forschungsbasiert nachgewiesen wurde, dass das Wissen über Modelle der Allgemeinen Didaktik die Qualität der Unterrichtsplanung bedingt und dass diese wiederum der Qualität des unterrichtlichen Handelns und der Leistung der Schülerinnen und Schüler bestimmt. Oder anders herum gefragt: kann Unterricht nicht unabhängig vom Wissen über und die Nutzung von allgemeindidaktischen Modellen „gut“ geplant und mit hoher Prozessqualität durchgeführt werden? Kann im Berufs-

\footnotetext{
4 Im Einzelfall wird eine forschungsbasierte Absicherung von behaupteten Zusammenhängen von vornherein als irrelevant markiert, wenn die Unterrichtsplanung als ,Schlüsselstelle des Unterrichtens“ hervorgehoben wird, die ,über Erfolg oder Misserfolg entscheiden kann, auch wenn kein kausaler Zusammenhang besteht“" (Zierer et al. 2015, S. 376).
} 
Tab. 8 Kriterien zur Analyse von schriftlichen Unterrichtsplanungen. (Quelle: König et al. 2015, S. 386)

1. Beschreibung situativer Bedingungen (Lerngruppe)

1.1 Die Lerngruppe wird beschrieben

1.2 Unterschiede in der Lerngruppe hinsichtlich kognitiver Voraussetzungen werden beschrieben

1.3 Unterschiede in der Lerngruppe hinsichtlich motivationaler Voraussetzungen werden beschrieben

2. Beschreibung der Aufgabe

2.1 Die Aufgabenstellung wird beschrieben

2.2 Die Aufgabenstellung (nicht: Nutzung oder Bearbeitung durch die Schüler) erfolgt differenziert hinsichtlich kognitiver Unterschiede der Schüler

2.3 Die unter [2.2] vorgenommene Differenzierung der Aufgabenstellung wird begründet

3. Anwendung erbrachter Beschreibungen auf die Situation

3.1 Bisheriger spezifischer Lernstand der Schüler (gesamte Lerngruppe) hinsichtlich der für die Unterrichtsstunde gewählten Aufgaben wird beschrieben - im Sinne des „nächsten Lernschrittes“

3.2 Bisheriger spezifischer Lernstand der Schüler (Differenzierung auf einzelne Schüler oder Schülergruppen) hinsichtlich der für die Unterrichtsstunde gewählten Aufgaben wird beschrieben - im Sinne des jeweiligen „,nächsten Lernschrittes“

4. Verknüpfung von Elementen der Planung

4.1 Aufgabenstellung und Ziel der Unterrichtsstunde werden verknüpft

4.2 Gruppierung der Schüler (grouping students) wird verknüpft mit der Differenzierung von Aufgaben

4.3 Die durch die Aufgabenstellung erzielten Ergebnisse werden kontrolliert

alltag womöglich ein kaum eingehend geplanter Unterricht auf der Prozess- und Ertragsebene trotzdem lernförderlich sein?

Während in den vorhergehenden Abschnitten bereits die jeweils in Rede stehenden Modellierungen und Operationalisierungen (an)diskutiert wurden, werden abschließend forschungsperspektivisch drei darüber hinaus gehende Herausforderungen aufgegriffen.

1. Qualitätskriterien für Unterrichtsplanungskompetenz und-performanz: Die Diagnose von König et al. (2015, S. 377), dass ein geteiltes Konzept der Planungskompetenz in der Literatur nicht erkennbar sei, kann sechs Jahre nach ihrer Formulierung trotz der Konjunktur der Forschung zur Unterrichtsplanung immer noch als zeitgemäß beschrieben werden. Damit einher geht die offene Frage, wie fachübergreifend eine ,gute“ Unterrichtsplanung, wie eine qualitätsvolle Planungsperformanz empirisch zu identifizieren und bestimmen ist, die auf die Ausprägung der ihr zugrunde liegenden Planungskompetenz schließen lässt. Sowohl das tradierte allgemeindidaktische Wissen samt der im Abschn. 1 genannten allgemeindidaktischen Grundprinzipien und der einschlägigen Modelle als auch die Befunde der empirischen Unterrichtsforschung, die bspw. übereinstimmend mit den Grundannahmen Allgemeiner Didaktik die Bedeutung der Adaptivität des Lernangebots betonen, dienen als Quelle für die Bestimmung von Inhaltsbereichen und als Indikatoren sowie Qualitätskriterien für die Analyse der Unterrichtsplanung. Diese Bezüge verweisen perspektivisch auf die Notwendigkeit ihrer empirischen Überprüfung, sodass die forschungsbasierte Legitimation notwendiger Qualitätskriterien für Planungsperformanz als Forschungsdesiderat ausgewiesen werden kann, das untrennbar mit der Operationalisierung von allgemeindidaktischer Planungs- 
kompetenz und hier ebenfalls mit ihrer empirisch abgesicherten Begründung verbunden ist. König et al. (2015, S. 396) können hier erste Befunde berichten und belegen, dass höhere Werte der von ihnen bei Referendarinnen und Referendaren erfassten Planungskompetenz (s. Tab. 8) mit einer besseren Bewertung des Qualitätsaspekts „Binnendifferenzierung“ durch die unterrichteten Schülerinnen und Schüler einhergehen.

Die Forderung nach einer forschungsbasierten Bestimmung und Begründung von Qualitätskriterien für Unterrichtsplanung(sperformanz) ist jedoch, das soll keineswegs unterschlagen werden, - gerade auch gemessen am Kriterium der Qualität des unterrichtlichen Handelns der Lehrkraft und der Prozessqualität des durchgeführten Unterrichts - womöglich viel leichter zu formulieren als zu realisieren. Verläuft ein Unterricht nach einem wohl begründeten, allen Grundprinzipien der Allgemeinen Didaktik folgenden Plan, so ist das keinesfalls zwingend Ausweis seiner Qualität (Gassmann 2013), im Gegenteil: es kann gerade der Unterricht eine höhere Qualität aufweisen, in dessen Verlauf der detailliert und vorbildlich ausgearbeitete Plan frühzeitig und vollständig über den Haufen geworfen wird. Angesichts der Intransparenz bezogen auf die nicht vollständig bekannten oder durchschaubaren wechselseitigen Beziehungen aller Planungsparameter und Wirkungen, der Dynamik der zu planende Situation, der Komplexität und Vernetzung der Planungsvariablen sowie der Koexistenz divergierender Ziele (Weingarten 2019, S. 19f.) vollzieht sich die Unterrichtsdurchführung generell eher zwischen dem im Plan Diagnostizierten, Antizipierten und Beabsichtigten und der situationsangemessenen Adaption oder gar Improvisation. Der Ansatz, die Bewertung der Qualität der Planungsperformanz an das Kriterium der Prozessqualität des realisierten Unterrichtsgeschehens zu binden, erweist sich somit als schwierig. Eine Entkopplung erscheint jedoch ebenso wenig denkbar, ist die Unterrichtsplanung doch kein Selbstzweck und die praktische Relevanz einer minuziösen Detailplanung im Sinne einer „Überplanung“ in Abgrenzung zu einer „Unterplanung“, einem „Adhocismus" (Weingarten 2019, S. 21) anhand der Planungsperformanz allein nicht zu bestimmen.

2. Zusammenhang der Wissensdimensionen und der Planungsperformanz: Der Bezug auf vorhandene Wissensbestände ist gerade bei der Unterrichtsplanung als kognitivem Vorgang (Bromme und Seeger 1979) im Gegensatz etwa zum unmittelbaren und schnellen Interaktionsgeschehen im Unterricht relevant, kann gerade hier entlastet vom unmittelbaren Handlungsdruck wohlüberlegt auf verschiedene Wissensbestände reflektiert zurückgegriffen werden (vgl. Wolf 2021). Insofern kann berechtigt, wie in den Abschn. 2 und 3 dargelegt, davon ausgegangen werden, dass Lehrkräfte auf verschiedene Facetten ihres Professionswissens zurückgreifen (Scholl et al. 2019). Unbestimmt ist jedoch, welche Bedeutung dabei welchen Teilen des Professionswissen genau zukommt und in welcher Beziehung die Wissensfacetten zueinander stehen. Behauptungen, dass sich im Planen von Unterricht „pädagogisches Wissen, Fachwissen und fachdidaktisches Wissen“ verbinden (Zierer et al. 2015, S. 375) oder gar, dass je fundierter das Professionswissen sei (fachlich, fachdidaktisch, pädagogisch), ,desto ausgeprägter zeigt sich die Planungskompetenz" (Zierer et al. 2015, S. 378), oder dass sich in der Unterrichtsplanung Fachkompetenz, didaktische Kompetenz und pädagogische Kompetenz 
manifestieren (Wernke und Zierer 2017, S. 10; Schmaltz 2019, S. 91), entbehren bislang forschungsbasierter empirischer Belege. Tatsächlich muss die angenommene Bedeutung des hier in Anschlag gebrachten Professionswissens vor dem Hintergrund erster Befunde womöglich in Teilen relativiert, in jedem Falle differenziert werden.

So haben Schröder et al. (2020; vgl. Vogelsang et al. 2019) die Fähigkeit zur Unterrichtsplanung von Lehramtsstudierenden im Fach Physik mittels eines Performanztests bei Lehramtsstudierenden im Praxissemester gemessen (Performanz ist hier die schriftliche Unterrichtsplanung) und die Zusammenhänge zwischen der Unterrichtsplanungsperformanz und dem Fachwissen, dem Fachdidaktischen Wissen und dem Erziehungswissenschaftlichen Wissen überprüft (Tab. 9). Offenbar manifestiert sich in der Unterrichtsplanung nicht, wie angenommen, die Fachkompetenz - hier festgemacht am getesteten physikalischen Fachwissen. Der Zusammenhang zwischen Fachdidaktischem und Erziehungswissenschaftlichem Wissen auf der einen und der Planungskompetenz auf der anderen Seite zeigt einen mittleren Effekt.

König et al. (2020a) haben die Bedeutung des getesteten Pädagogischen Wissens für die Planungsperformanz im Unterrichtsfach Deutsch von Referendarinnen und Referendaren festgemacht an der pädagogischen Adaptivität als situationsspezifischer Anforderung in schriftlichen Unterrichtsplanungen überprüft. Im Ergebnis korreliert das Pädagogische Wissen (Gesamtscore) nicht mit der Adaptivität in schriftlichen Unterrichtsplanungen ( $r=0,02$, n. s.). Lediglich die mit dem Kriterium korrespondierende Subdimension des Wissenstest zur Adaptivität steht in einem signifikanten Zusammenhang mit der Adaptivität in der schriftlichen Unterrichtsplanung $(r=0,21)$ bzw. bedingt diese $(\beta=0,20)$ (König et al. 2020a).

Die Befunde verweisen erneut darauf, dass die im Diskurs formulierten Annahmen in Gestalt von Gewissheiten zunächst mit Vorsicht zu genießen sind. Sie markieren zudem, dass die Forschung zur Bedeutung und Wirkung des Professionswissens für Unterrichtsplanungskompetenz und -performanz noch vor vielfältigen Herausforderungen steht, zu denen auch die Fragen nach den Zusammenhängen zwischen Fachwissen (Unterrichtsplanung ohne Fachwissen?) und fachdidaktischem Wissen sowie fachdidaktischem Wissen und pädagogischem Wissen zählen. Angesichts des wiederholten Verweises auf die Bedeutung des Professionswissens erscheint es jedoch vorranging geboten zu bestimmen, welche Wissensfacetten es konkret sind, die etwa als Teil des pädagogischen Wissens Unterrichtsplanungskompetenz bedingen. Das allgemeine und damit unspezifische, allzu abstrakte deklarative Wissen über allgemeindidaktische Modelle oder Klafkis Allgemeinbildungsverständnis dürfte es vermutlich nicht sein. Stattdessen verspricht ein fokussierter Zugang, wie er sich in den Operationalisierungen von König et al. (2020a) zeigt, für zukünftige Forschungsanstrengungen geeigneter zu sein.

3. Unterrichtsplanungskompetenz als Phänomen der Lehrer*innenbildung: Unabhängig von der methodischen Anlage bisheriger Untersuchungen und der ihnen zugrunde liegenden Modellierungen haben diese eines gemein: befragt, getestet oder zur Abfassung bzw. Erläuterung von schriftlichen Unterrichtsplanungen mit anschließender Auswertung angehalten werden Lehramtsstudierende in der ersten Phase der Lehrer*innenbildung und - im Vergleich seltener - Referendarinnen 
und Referendare in der zweiten Phase. Wenn aber die von Sandfuchs im Handbuch Unterricht (2006a, S. 687) formulierte und durch die Befunde der Expertiseforschung (vgl. Munthe und Conway 2017) gestützte Annahme eines lang andauernden Entwicklungsprozesses unterrichtlicher Planungskompetenz in der Berufsbiographie insbesondere über die erste, aber auch über die zweite Phase der Lehrer*innenbildung hinaus zutrifft, dann stellt sich die Frage, was in der vor allem auf die erste Phase der Lehrer*innenbildung fokussierten Forschung überhaupt erfasst wird bzw. erfasst werden kann. Eine voll ausgebildete Planungskompetenz wird auch am Ende der Lehrer*innenbildung (2. Phase) nicht erwartet werden können - es sei denn, es würde doch, wie eingangs angemerkt, von einer genialischen, intuitiven Fertigkeit (vgl. Busch 2009) ausgegangen. Wird also in der Forschung zur Unterrichtsplanungskompetenz in der Lehrer*innenbildung ein Konstrukt empirisch erhoben, das zu diesem Zeitpunkt noch gar nicht zu erwarten und trotzdem etwa in Selbstauskünften von Lehramtsstudierenden hoch ausgeprägt ist? Was sagt dies dann über die Validität der Skalen zur selbsteingeschätzten Planungskompetenz aus, wenn Lehramtsstudierende bereits angeben, (gut oder sehr gut) zu können, was erst noch erlernt und in einem langwierigen Professionalisierungsprozess auszubilden ist (vgl. Rothland 2018b)?

Wenn unterrichtliche Planungskompetenz modelliert, operationalisiert und empirisch erfasst wird, dann wäre - so der generelle Einwand - zuerst bei denen anzusetzen, die über die hier im Fokus stehende Kompetenz bereits in unterschiedlicher Ausprägung verfügen (können): den im Beruf stehenden, routinierten Lehrerinnen und Lehrern. Mit der Modellierung, Operationalisierung und empirischen Erfassung von Lesekompetenz würde auch nicht im Kindergarten oder in der ersten Klasse angesetzt. Anders: Unterrichtsplanungskompetenz als „neues“ Konstrukt lässt sich womöglich besser in potenziell voller Ausprägung identifizieren und empirisch erfassen als im Entstehen, in der Entwicklung.

Nun gibt es ohne Zweifel nachvollziehbare, auch forschungspragmatische Gründe dafür, die Lehrer*innenbildung in den Blick zu nehmen. Ohne den Vergleich mit den berufserfahrenen Lehrkräften bleibt die unterrichtliche Planungskompetenz aber eigentümlich unbestimmt. Zudem ergeben sich aus der Konzentration einer prosperierenden Forschung zur Unterrichtsplanungskompetenz auf die (erste Phase der) Lehrer*innenbildung problematische Parallelen: Allgemeindidaktische Planungsmodelle erscheinen in der bisherigen Forschung schließlich ebenfalls als Phänomene der Lehrer*innenbildung und nicht der Berufspraxis (vgl. Bromme 1981; Oehlschläger 1978; Haas 1998; Seel 1997), so wie auch ausführliche Un-

Tab. 9 Bivariate Korrelationen von Fachwissen $(F W)$, Fachdidaktischem Wissen $(F W)$ Erziehungswissenschaftlichem Wissen $(E W)$ und der Unterrichtsplanungsperformanz von Lehramtsstudierenden im Fach Physik getrennt nach Messzeitpunkten zu Beginn und am Ende des Praxissemesters (PS). (Schröder et al. 2020, S. 117)

\begin{tabular}{llllllll}
\hline & & FW & & FDW & \multicolumn{3}{c}{ EW } \\
& & $r$ & $n$ & $r$ & $n$ & $r$ & $n$ \\
\hline Planung & Beginn PS & 0,01 (n.s.) & 59 & $0,32^{* *}$ & 74 & $0,36^{* *}$ & 55 \\
& Ende PS & $-0,06$ (n.s.) & 38 & $0,33^{* *}$ & 71 & $0,25^{*}$ & 71 \\
\hline
\end{tabular}

$* *=p<.01, *=p<.05$ 
terrichtsplanungen im Sinne elaborierter schriftlicher Stundenvorbereitungen generell als Ausbildungsartefakte beschrieben werden (Seel 2011). Lehrerinnen und Lehrer, die im Beruf angekommen sind, planen ihren Unterricht mit geringerem zeitlichen Aufwand nicht mehr so, wie sie es im Rahmen der Lehrer*innenbildung „gelernt" und mit hohem Zeitaufwand praktiziert haben (Munthe und Conway 2017). Sie lassen sich auch kaum von allgemeindidaktischen Modellen leiten und verfassen keine detaillierten Unterrichtsentwürfe, wie sie im Referendariat produziert werden. Das führt aber - zum Glück - nicht dazu, dass sie keinen ,guten“ Unterricht planen bzw. durchführen könnten.

Funding Open Access funding enabled and organized by Projekt DEAL.

Open Access Dieser Artikel wird unter der Creative Commons Namensnennung 4.0 International Lizenz veröffentlicht, welche die Nutzung, Vervielfältigung, Bearbeitung, Verbreitung und Wiedergabe in jeglichem Medium und Format erlaubt, sofern Sie den/die ursprünglichen Autor(en) und die Quelle ordnungsgemäß nennen, einen Link zur Creative Commons Lizenz beifügen und angeben, ob Änderungen vorgenommen wurden.

Die in diesem Artikel enthaltenen Bilder und sonstiges Drittmaterial unterliegen ebenfalls der genannten Creative Commons Lizenz, sofern sich aus der Abbildungslegende nichts anderes ergibt. Sofern das betreffende Material nicht unter der genannten Creative Commons Lizenz steht und die betreffende Handlung nicht nach gesetzlichen Vorschriften erlaubt ist, ist für die oben aufgeführten Weiterverwendungen des Materials die Einwilligung des jeweiligen Rechteinhabers einzuholen.

Weitere Details zur Lizenz entnehmen Sie bitte der Lizenzinformation auf http://creativecommons.org/ licenses/by/4.0/deed.de.

\section{Literatur}

Adl-Amini, B., Künzli, R. (Hrsg.). (1980). Didaktische Modelle und Unterrichtsplanung. München: Juventa.

Arnold, K.-H., Schüle, C., Besa, K.-S., Bach, A. (2017). Test zur Erfassung allgemeindidaktischen Wissens zur Planung, Analyse und Durchführung von Unterricht (UPDA-T): Skalenhandbuch. Hildesheim: Universität, Institut für Erziehungswissenschaft.

Bach, A. (2013). Kompetenzentwicklung im Schulpraktikum. Münster: Waxmann.

Bach, A., Besa, K.-S., Brodhäcker, S., Arnold, K.-H. (2012). Kompetenzentwicklung in Schulpraktika: Erfassung allgemeindidaktischer Kompetenz zur Planung, Durchführung und Analyse von Unterricht. In T. Hascher G. H. Neuweg (Hrsg.), Forschung zur (Wirksamkeit der) Lehrerbildung (S. 105-122). Wien: LIT.

Bach, A., Brodhäcker, S., Arnold, K.-H. (2010). Entwicklung allgemeindidaktischer Kompetenz in Schulpraktika: Erfassung der Kompetenzen zur Unterrichtsplanung, -durchführung und -analyse. Lehrerbildung auf dem Prüfstand, 3(2), 158-178.

Backhaus, S. (2018). Planungsaspekte erfahrener Lehrkräfte im Schuldienst (PerLe). Eine empirische Studie. Berlin: Logos.

Baer, M., Kocher, M., Wyss, C., Guldimann, T., Larcher, S., Dörr, G. (2011). Lehrerbildung und Praxiserfahrung im ersten Berufsjahr und ihre Wirkung auf die Unterrichtskompetenzen von Studierenden und jungen Lehrpersonen im Berufseinstieg. Zeitschrift für Erziehungswissenschaft, 14(1), 85-117.

Baumert, J., Kunter, M. (2006). Stichwort: Professionelle Kompetenz von Lehrkräften. Zeitschrift für Erziehungswissenschaft, 9, 469-520.

Baumert, J., Kunter, M. (2011). Das Kompetenzmodell von COACTIV. In M. Kunter, J. Baumert, W. Blum, U. Klusmann, S. Krauss M. M. Neubrand (Hrsg.), Professionelle Kompetenz von Lehrkräften. Ergebnisse des Forschungsprogramms COACTIV (S. 29-53). Münster: Waxmann. 
Beck, E., Baer, M., Guldimann, T., Bischoff, S., Brühwiler, C. (2008). Adaptive Lehrkompetenz: Analyse und Struktur, Veränderbarkeit und Wirkung handlungssteuernden Lehrerwissens. Münster: Waxmann.

Blömeke, S., Gustafsoon, J.-E., Shavelson, R. (2015). Beyond dichotomies: viewing competence as a continuum. Zeitschrift für Psychologie, 223, 3-13.

Bohl, T. (2004). Empirische Unterrichtsforschung und Allgemeine Didaktik. Ein prekäres Spannungsverhältnis und Konsequenzen im Kontext der PISA-Studie. Die Deutsche Schule, 96, 414-425.

Bromme, R. (1981). Die alltägliche Unterrichtsvorbereitung von Mathematiklehrern. Unterrichtswissenschaft, 8, 142-156.

Bromme, R., Seeger, F. (1979). Unterrichtsplanung als Handlungsplanung. Eine psychologische Einführung in die Unterrichtsvorbereitung. Königstein: Scriptor.

Busch, M. (2009). Anleitungen zur Unterrichtsplanung in sozialwissenschaftlichen Fächern. Bereichsrezension aktueller fachdidaktischer Planungskonzepte. Journal of Social Science Education, 8(2), 124-145.

Frey, A. (2014). Kompetenzmodelle und Standards in der Lehrerbildung und im Lehrerberuf. In E. Terhart, H. Bennewitz M. Rothland (Hrsg.), Handbuch der Forschung zum Lehrerberuf (2. Aufl. S. 712-744). Münster: Waxmann.

Gassmann, C. (2013). Erlebte Aufgabenschwierigkeit bei der Unterrichtsplanung. Eine qualitativ-inhaltsanalytische Studie zu den Praktikumsphasen der universitären Lehrerbildung. Wiesbaden: Springer VS.

Glutsch, N., Bremerich-Vos, A., Buchholtz, C., König, J., Fladung, I., Lammerding, S., Strauß, S., \& Schleiffer, C. (2019). PlanvoLL-D - Die Bedeutung des professionellen Wissens angehender Deutschlehrkräfte für ihre Planung von Unterricht: Validierung und methodische Innovation. Skalendokumentation zu Instrumenten der Ausbildungsinhalte und Schulpraxis, Messzeitpunkte 1 und 2, Sommer 2016 und Winter 2017/18. Köln: Universität zu Köln.

Gräsel, C. (2006). Das Verhältnis von Erziehungswissenschaft und Bildungsforschung aus der Perspektive der Unterrichtsforschung. In H. Merkens (Hrsg.), Erziehungswissenschaft und Bildungsforschung (S. 97-108). Wiesbaden: VS.

Haag, L., Kiel, E., Trautmann, M. (2015). Einführung in den Thementeil „Klassenmanagement/Klassenführung - Perspektiven, Befunde, Kontroversen. In L. Haag, E. Kiel M. Trautmann (Hrsg.), Klassenmanagement/Klassenführung - Perspektiven, Befunde, Kontroversen. Jahrbuch für Allgemeine Didaktik, (Bd. 5, S. 9-14). Baltmannsweiler: Schneider Verlag Hohengehren.

Haas, A. (1998). Unterrichtsplanung im Alltag. Eine empirische Untersuchung zum Planungshandeln von Hauptschul-. Realschul- und Gymnasiallehrern. Regensburg: Roderer.

Heimann, P., Otto, G., Schulz, W. (1965). Unterricht - Analyse und Planung. Hannover: Schroedel.

Hohenstein, F., Zimmermann, F., Kleickmann, T., Köller, O., Möller, J. (2014). Sind die bildungswissenschaftlichen Standards für die Lehramtsausbildung in den Curricula der Hochschulen angekommen? Zeitschrift für Erziehungswissenschaft, 17, 497-507.

Jäger, S., Maier, U. (2019). Unterrichtsplanung. In E. Kiel, B. Herzig, U. Maier U. Sandfuchs (Hrsg.), Handbuch Unterrichten an allgemeinbildenden Schulen (S. 455-466). Bad Heilbrunn: Klinkhardt/ UTB.

Kiel, E., Haag, L., Keller-Schneider, M., Zierer, K. (2014). Unterricht planen, durchführen, reflektieren. Berlin: Cornelsen.

Kiper, H., Mischke, W. (2009). Unterrichtsplanung. Weinheim, Basel: Beltz.

Klafki, W. (1958). Didaktische Analyse als Kern der Unterrichtsvorbereitung. Die Deutsche Schule, 50, 450-463.

Klafki, W. (1980). Zur Unterrichtsplanung im Sinne kritisch-konstruktiver Didaktik. In B. Adl-Amini \& R. Künzli (Hrsg.), Didaktische Modelle der Unterrichtsplanung (S. 11-48). Weinheim: Juventa.

KMK = Kultusministerkonferenz (2019). Standards für die Lehrerbildung: Bildungswissenschaften. Berlin/Bonn: KMK. Beschluss der Kultusministerkonferenz vom 16.12.2004 i.d. F. vom 16.05.2019

König, J., Buchholtz, C., Dohmen, D. (2015). Analyse von schriftlichen Unterrichtsplanungen: Empirische Befunde zur didaktischen Adaptivität als Aspekt der Planungskompetenz angehender Lehrkräfte. Zeitschrift für Erziehungswissenschaft, 18, 375-404.

König, J., Bremerich-Vos, A., Buchholtz, C., Lammerding, S., Strauß, S., Fladung, I., Schleiffer, C. (2017a). Die Bedeutung des Professionswissens von Referendarinnen und Referendaren mit Fach Deutsch für ihre Planungs-kompetenz (PlanvoLL-D). In S. Wernke K. Zierer (Hrsg.), Die Unterrichtsplanung: Ein in Vergessenheit geratener Kompetenzbereich?! (S. 121-133). Bad Heilbrunn: Klinkhardt. 
König, J., Bremerich-Vos, A., Buchholtz, C., Lammerding, S., Strauß, S., Fladung, I., Schleiffer, C. (2017b). Modelling and validating the learning opportunities of preservice language teachers: on the key components of the curriculum for teacher education. European Journal of Teacher Education, 40(3), 394-412.

König, J., Bremerich-Vos, A., Buchholtz, C., Glutsch, N. (2020a). General pedagogical knowledge, pedagogical adaptivity in written lesson plans, and instructional practice among preservice teachers. Journal of Curriculum Studies, 52(6), 800-822.

König, J., Bremerich-Vos, A., Buchholtz, C., Fladung, I., Glutsch, N. (2020b). Pre-service teachers' generic and subject-specific lesson-planning skills: on learning adaptive teaching during initial teacher education. European Journal of Teacher Education, 43(2), 131-150.

Kuchartz, D., Mackowiak, K., Fain, V. (2014). Entwicklung von Planungskompetenz im Berufseinstieg. journal für lehrerInnenbildung, 14(4), 20-24.

Larcher, S., Müller, P., Baer, M., Dörr, G., Edelmann, D., Guldimann, T., Kocher, M., Wyss, C. (2010). Unterrichtskompetenz über die Zeit. Unterrichten lernen zwischen Studienbeginn und Ende des ersten Berufsjahres. In J. Abel G. Faust (Hrsg.), Wirkt Lehrerbildung? Antworten aus der empirischen Forschung (S. 57-72). Münster: Waxmann.

Lohmann, V., Seidel, V., Terhart, E. (2011). Bildungswissenschaften in der universitären Lehrerbildung: Curriculare Strukturen und Verbindlichkeiten. Eine Analyse aktueller Studienordnungen an nordrhein-westfälischen Universitäten. Lehrerbildung auf dem Prüfstand, 4, 271-302.

Maag Merki, K. (2009). Kompetenz. In S. Andresen, R. Casale, T. Gabriel, R. Horlacher, S. Larcher Klee J. Oelkers (Hrsg.), Handwörterbuch Erziehungswissenschaft (S. 492-506). Weinheim: Beltz.

Munthe, E., Conway, P.F. (2017). Evolution of research on teachers' planning: implications for teacher education. In D. J. Clandinin J. Husu (Hrsg.), SAGE handbook of research on teacher education (S. 836-852). Thousand Oaks: SAGE.

Nauck, J. (1996). Zur Planungs- und Reflexionskompetenz von Studierenden. Inhaltsanalyse didaktischer Akten zum Allgemeinen Schulpraktikum. In K.-H. Sander (Hrsg.), Schulpraktische Studien. Erfahrungen mit dem Braunschweiger Modell der Lehrerausbildung (S. 181-210). Braunschweig: Schmidt.

Oehlschläger, H. J. (1978). Zur Praxisrelevanz pädagogischer Literatur. Strukturen und Trends zur Literaturrezeption praktizierender Lehrer. Stuttgart: Klett-Cotta.

Peterßen, W.H. (2006). Handbuch Unterrichtsplanung (9. Aufl.). München: Oldenbourg. Nachdr.

Rey, T., Lohse-Bossenz, H., Wacker, A., Heyl, V. (2018). Adaptive Planungskompetenz bei angehenden Lehrkräften in der zweiten Phase der Lehrerbildung. heiEDUCATION, 1(2), 127-150.

Rothland, M. (2013). Wiederbelebung einer Totgesagten. Anmerkungen zur Reanimation der Allgemeinen Didaktik. Zeitschrift für Erziehungswissenschaft, 16(3), 629-645.

Rothland, M. (2018a). Allgemeine Didaktik und empirische Unterrichtsforschung als Teilgebiete der Schulpädagogik. Die Deutsche Schule, 110, 369-382.

Rothland, M. (2018b). Yes, we can! Anmerkungen zur trügerischen „Kompetenzentwicklung“ von Lehramtsstudierenden im Praxissemester. Beiträge zur Lehrerinnen- und Lehrerbildung, 36(3), 482-495.

Sandfuchs, U. (2006a). Grundfragen der Unterrichtsplanung. In K.-H. Arnold, U. Sandfuchs J. Wiechmann (Hrsg.), Handbuch Unterricht (S. 685-694). Bad Heilbrunn: Klinkhardt.

Sandfuchs, U. (2006b). Ebenen, Prinzipien und Situationen der Planung. In K.-H. Arnold, U. Sandfuchs J. Wiechmann (Hrsg.), Handbuch Unterricht (S. 695-701). Bad Heilbrunn: Klinkhardt.

Schmaltz, C. (2019). Heterogenität als Herausforderung für die Professionalisierung von Lehrkräften. Wiesbaden: Springer VS.

Schnebel, S., Kreis, A. (2014). Kollegiales Unterrichtscoaching zwischen Lehramtsstudierenden. journal für lehrerInnenbildung, 14(4), 41-46.

Schnebel, S., Kreis, A., Musow, S. (2017). Wie schätzen Studierende ihre Planungskompetenz und den Nutzen kooperativer Unterrichtsplanung ein? In A. Kreis S. Schnebel (Hrsg.), Peer Coaching in der praxissituierten Ausbildung von Lehrpersonen. Sonderheft der Lehrerbildung auf dem Prüfstand. (S. 107-128). Landau: Verlag Empirische Pädagogik.

Scholl, D., Küth, S., Flath, M., Hertema, H., Schwarz, B., Wolters, P., Rheinländer, K., Schüle, C. (2019). Zum Konstrukt der Planungskompetenz in allgemein- und fachdidaktischen Ansätzen. In D. Scholl, S. Wernke D. Behrens (Hrsg.), Jahrbuch für Allgemeine Didaktik (S. 75-94). Baltmannsweiler: Schneider Verlag Hohengehren.

Schröder, J., Riese, J., Vogelsang, C., Borowski, A., Buschhüter, D., Enkrott, P., Kempin, M., Kulgemeyer, C., Reinhold, P., Schecker, H. (2020). Die Messung der Fähigkeit zur Unterrichtsplanung imFach Physik mit Hilfe eines standardisierten Performanztests. Zeitschrift für Didaktik der Naturwissenschaften, 26, 103-122. 
Schüle, C., Besa, K.-S., Arnold, K.-H. (2017). Entwicklung und Validierung eines Testinstruments zur Erfassung allgemeindidaktischer Kompetenz. In S. Wernke K. Zierer (Hrsg.), Die Unterrichtsplanung: Ein in Vergessenheit geratener Kompetenzbereich?! (S. 17-31). Bad Heilbrunn: Klinkhardt.

Schüle, C., Besa, K.-S., Bach, A., Arnold, K.-H. (2019). Entwicklung und Validierung eines Wissenstests zur Erfassung allgemeindidaktischer Planungskompetenz. In Symposium Modellierung und Messung der unterrichtlichen Planungskompetenz auf der Jahrestagung der AEPF/KBBB 2019. Münster. Vortrag.

Schulze-Stocker, F. (2016). Die Normierung der Bildungswissenschaften in den Lehramtsstudiengängen durch gesetzliche Vorgaben: Wie (re)organisieren Universitäten ihr bildungswissenschaftliches Lehrangebot? Münster: Monsenstein und Vannerdat.

Seel, A. (1997). Von der Unterrichtsplanung zum konkreten Lehrerhandeln - Eine Untersuchung zum Zusammenhang von Planung und Durchführung von Unterricht bei Hauptschullehrerstudentinnen. Unterrichtswissenschaft, 25, 257-273.

Seel, A. (2011). Wie angehende Lehrer/innen das Planen lernen. Empirische Befunde zur ausbildungsbezogenen Unterrichtsplanung. In K. Zierer (Hrsg.), Entwicklung und Weiterentwicklung allgemeindidaktischer Modelle der Unterrichtsplanung. Jahrbuch für Allgemeine Didaktik. (S. 31-45). Baltmannsweiler: Schneider Verlag Hohengehren.

Seel, A., Aprea, C. (2014). Editorial. journal für lehrerInnenbildung, 14(4), 4-6.

Shavelson, R. J. (1973). What is the basic teaching skill? Journal of Teacher Education, 24, 144-151.

Standop, J., Jürgens, E. (2015). Unterricht planen, gestalten und evaluieren. Bad Heilbrunn: Klinkhardt/UTB.

Vogelsang, C., Riese, J. (2017). Wann ist eine Unterrichtsplanung ,gut‘? Planungsperformanz in Praxisratgebern zur Unterrichtsplanung. In S. Wernke K. Zierer (Hrsg.), Die Unterrichtsplanung: Ein in Vergessenheit geratener Kompetenzbereich?! (S. 47-61). Bad Heilbrunn: Klinkhardt.

Vogelsang, C., Borowski, A., Buschhüter, D., Enkrott, P., Kempin, M., Kulgemeyer, C., Reinhold, P., Riese, J., Schecker, H., Schröder, J. (2019). Entwicklung von Professionswissen und Unterrichtsperformanz im Lehramtsstudium Physik - Analysen zu valider Testwertinterpretation. Zeitschrift für Pädagogik, 65(4), 473-491.

Voss, T., Kunina-Habenicht, O., Hoehne, V., Kunter, M. (2015). Stichwort Pädagogisches Wissen von Lehrkräften. Empirische Zugänge und Befunde. Zeitschrift für Erziehungswissenschaft, 18(2), 187-223.

Weingarten, J. (2019). Wie planen angehende Lehrkräfte ihren Unterricht? Empirische Analysen zur kompetenzorientierten Gestaltung von Lernangeboten. Münster: Waxmann.

Werner, J. (2017). Allgemeindidaktische Entwicklungen bei der Unterrichtsplanung von Lehramtsstudierenden im Rahmen des Praxismoduls „Allgemeines Schulpraktikum“. Baltmannsweiler: Schneider Verlag Hohengehren.

Wernke, S., Zierer, K. (2017). Die Unterrichtsplanung. Ein in Vergessenheit geratener Kompetenzbereich? In S. Wernke K. Zierer (Hrsg.), Die Unterrichtsplanung: Ein in Vergessenheit geratener Kompetenzbereich?! (S. 7-16). Bad Heilbrunn: Klinkhardt.

Westerman, D. A. (1991). Expert and novice teacher decision making. Journal of Teacher Education, 42(4), 292-305.

Wiater, W. (2006). Didaktische Theoriemodelle und Unterrichtsplanung. In K.-H. Arnold, U. Sandfuchs J. Wiechmann (Hrsg.), Handbuch Unterricht (S. 675-685). Bad Heilbrunn: Klinkhardt.

Wolf, J. (2020). (Wie) Wirkt Lehrerbildung? Ein Beitrag zur geschichtsdidaktischen Lehrerkompetenz- und Lehrerbildungswirksamkeitsforschung am Beispiel des Unterrichtsplanungswissens von angehenden GeschichtslehrerInnen. Dissertation, Fakultät für Geschichtswissenschaft. Bochum: Ruhr-Universität Bochum.

Wolf, J. (2021). Geschichtsunterricht planen können. Geschichte in Wissenschaft und Unterricht, 72, $58-74$.

Yinger, R. J. (1980). A study of teacher planning: description and a model of preactive decision making. The Elementary School Journal, 80(3), 107-127.

Ziegelbauer, C., Ziegelbauer, S. (2019). Unterrichtsplanung. In M. Gläser-Zikuda, M. Harring C. Rohlfs (Hrsg.), Handbuch Schulpädagogik (S. 427-493). Münster: Waxmann.

Zierer, K., Werner, J., Wernke, S. (2015). Besser planen? Mit Modell! Empirisch basierte Überlegungen zur Entwicklung eines Planungskompetenzmodells. Die Deutsche Schule, 107, 375-395. 